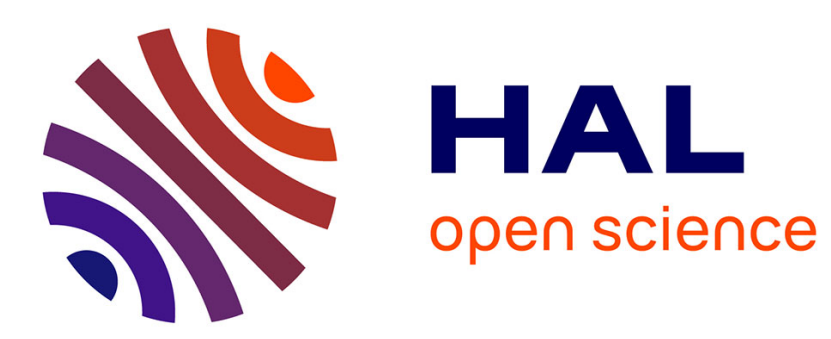

\title{
The Iso-Level Scheduling Heuristic for Heterogeneous Processors
}

Olivier Beaumont, Vincent Boudet, Yves Robert

\section{To cite this version:}

Olivier Beaumont, Vincent Boudet, Yves Robert. The Iso-Level Scheduling Heuristic for Heterogeneous Processors. [Research Report] LIP RR-2001-22, Laboratoire de l'informatique du parallélisme. 2001, 2+24p. hal-02101928

\section{HAL Id: hal-02101928 \\ https://hal-lara.archives-ouvertes.fr/hal-02101928}

Submitted on 17 Apr 2019

HAL is a multi-disciplinary open access archive for the deposit and dissemination of scientific research documents, whether they are published or not. The documents may come from teaching and research institutions in France or abroad, or from public or private research centers.
L'archive ouverte pluridisciplinaire HAL, est destinée au dépôt et à la diffusion de documents scientifiques de niveau recherche, publiés ou non, émanant des établissements d'enseignement et de recherche français ou étrangers, des laboratoires publics ou privés. 


\section{Laboratoire de l'Informatique du Parallélisme}

École Normale Supérieure de Lyon

Unité Mixte de Recherche CNRS-INRIA-ENS LYON no 5668

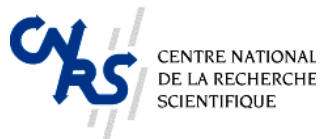

\section{The Iso-Level Scheduling Heuristic for Heterogeneous Processors}

Olivier Beaumont

Vincent Boudet

May 2001

Yves Robert

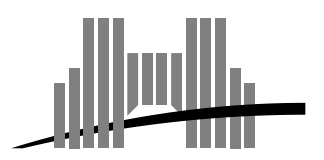

École Normale Supérieure de Lyon

46 Allée d'Italie, 69364 Lyon Cedex 07, France

Téléphone : $+33(0) 4.72 .72 .80 .37$

Télécopieur : +33(0)4.72.72.80.80

Adresse électronique : lip@ens-lyon.fr 


\title{
The Iso-Level Scheduling Heuristic for Heterogeneous Processors
}

\author{
Olivier Beaumont \\ Vincent Boudet \\ Yves Robert
}

May 2001

\begin{abstract}
Scheduling computational tasks on processors is a key issue for highperformance computing. Although a large number of scheduling heuristics have been presented in the literature, most of them target only homogeneous resources. We present a new scheduling heuristic for heterogeneous processors, which improves the load-balancing achieved at each decision step while keeping a low complexity. Experimental comparisons with five heuristics taken from the literature (BIL, GDL, CPOP, HEFT and PCT) and using six classical testbeds, show very favorable results.
\end{abstract}

Keywords: heterogeneous processors, unrelated parallel machines, scheduling heuristics, allocation, complexity.

\section{Résumé}

L'ordonnancement de graphes est un problème fondamental du calcul parallèle. Bien que de nombreuses heuristiques d'ordonnancement existent dans la littérature, la plupart d'entre elles ne s'intéressent qu'à des ressources homogènes. Nous présentons une nouvelle heuristique d'ordonnancement pour processeurs hétérogènes qui équilibre la charge de travail à chaque étape de décision tout en ayant une faible complexité. Des comparaisons expérimentales avec cinq heuristiques de la littérature (BIL, GDL, CPOP, HEFT et PCT) sur six problèmes classiques donnent de très bons résultats.

Mots-clés: processeurs hétérogènes, heuristiques d'ordonnancement, distribution, complexité. 


\section{Introduction}

The efficient scheduling of application tasks is critical to achieving high performance in parallel and distributed systems. The objective of scheduling if to find a mapping of the tasks onto the processors, and to order the execution of the tasks so that: (i) task precedence constraints are satisfied; and (ii) a minimum schedule length is provided. Since the scheduling problem with communication delays is NP-hard [3], various heuristics have been proposed in the literature (see the tutorial [1]).

Although various different approaches are used to solve the task scheduling problem, most of them target homogeneous processors only. Heterogeneity poses new challenges to scheduling techniques. Scheduling methods that are suitable for homogeneous environments may well not be efficient for heterogeneous domains. For instance, clustering techniques (such as Gerasoulis and Yang's dominant sequence clustering [10]) are widely used in the context of homogeneous parallel machines, while they seem difficult to use in the context of heterogeneous processors. In the literature, most heuristics for heterogeneous processors are adaptations of list-scheduling techniques for homogeneous processors, and are still based upon critical paths and bottom levels.

In this paper, we introduce a new scheduling heuristic for heterogeneous processors, which we name the Iso-Level Heterogeneous Allocation (ILHA) heuristic. In a word, the main characteristic of the ILHA heuristic is a better load-balancing at each decision step, which is achieved by considering a chunk of several ready tasks rather than a single one; the idea is to allocate to each processor a number of the tasks in the chunk that is proportional to its computing power. We compare the ILHA heuristic with five heuristics taken from the literature: the minimum Partial Completion Time static priority (PCT) heuristic, the Best Imaginary Level (BIL) heuristic, the Heterogeneous Earliest Finish Time (HEFT) heuristic, the Critical Path on a Processor (CPOP) heuristic and the Generalized Dynamic Level (GDL) heuristic. For the experimental comparisons, we use six classical testbeds: LAPLACE, LU, STENCIL, FORK-JOIN, DOOLITTLE, and LDMt. All these comparisons show very favorable results. Note that the ILHA heuristic requires a simple graph traversal, which renders it very attractive to process huge size problems.

The rest of the paper is organized as follows. Section 2 is devoted to some technical preliminaries (definitions and notations). We briefly survey the five heuristics from the literature in Section 3. We present the ILHA heuristic in Section 4. In Section 5, six classical testbeds are used to compare the different heuristics. Finally, we give some concluding remarks in Section 6.

\section{Preliminaries}

In this section, we specify some notations for the standard macro-dataflow model, which is widely used in the scheduling literature [1]. For each task scheduling algorithm, the input is a directed acyclic graph $\mathcal{G}=(\mathcal{N}, \mathcal{A})$, that models a parallel program, where $\mathcal{N}=\left\{N_{i}: i=1, \cdots, N\right\}$ is a set of $N$ nodes and $\mathcal{A}=\left\{A_{i, j}\right\}$ is a set of edges. A node in the DAG represents a task. Each task has a computation cost which is defined as the amount of computation cycles needed to process it. The time needed to compute this task on a processor is then the product of this computation cost by the cycle time of the processor. An edge corresponds to a precedence constraint and has a communication cost. Each edge $A_{i, j}$ carries a label $D_{i, j}$ which specifies the amount of data that $N_{i}$ passes to $N_{j}$. This can be used to compute the time needed to achieve the communication. We suppose that if both tasks are assigned to the same processor, there is no communication cost; otherwise, we pay a cost proportional to $D_{i, j}$, regardless the location of the processors. Moreover, we suppose that we can realize an unlimited number of communications simultaneously.

A task without any input edge is called an entry task while a task with no output edge is called an exit task. A task is said ready when all its predecessors have finished their execution. We denote by $\operatorname{Pred}\left(N_{i}\right)$ the set of the immediate predecessors of task $N_{i}$ and by $\operatorname{Succ}\left(N_{i}\right)$ the set of the immediate successors of task $N_{i}$.

The target architecture consists of a set of $p$ heterogeneous processors $\mathcal{P}=\left\{P_{1}, P_{2}, \ldots, P_{p}\right\}$ so that computation can be overlapped with communication and there is no limitation on the communication links: as soon as a task $N_{i}$ is completed, data $D_{i, j}$ is sent to all its successors. The execution time of node $N_{i}$ on processor $P_{j}$, given by $E\left(N_{i}, P_{j}\right)$ (denoted further by $e_{i, j}$ ) is available at compile time for each node-processor pair. 
Our scheduling objective is to minimize the scheduling length where all interprocessor communication overheads are included. This scheduling problem is NP-complete even if there is an infinite number of processors available [3], hence the need to rely on heuristics.

\section{The algorithms}

In this section we survey five heuristics from the literature.

\subsection{Minimum partial completion time static priority (PCT) algorithm}

Maheswaran and Siegel present in [5] a dynamic algorithm. Their heuristic refines a given mapping which has already been computed statically. It can be used from scratch to compute a static mapping at compile time by using any basic schedule as input (for example, assume that every task is allocated to the fastest processor). In the following, we assume that we already have a scheduling of our task graph. The first phase of the algorithm assigns ranks to each task. The second phase orders the tasks and uses a minimization criterion to solve the mapping problem.

Consider the first phase of the algorithm. We assign to each task a priority equals to an estimation of the time needed to finish the program. Since we already have a scheduling, we take communications into consideration. Letting $P_{j}$ be the processor to which task $N_{i}$ is assigned in the given scheduling, we define the priority as follows:

$$
\operatorname{priority}\left(N_{i}\right)=e_{i, j}+\max _{N_{k} \in \operatorname{Succ}\left(N_{i}\right)}\left(c_{i, k}+\operatorname{priority}\left(N_{k}\right)\right)
$$

where $c_{i, k}=D_{i, k} \times \tau$ is the time needed to send the data from the node $N_{i}$ to the node $N_{k}$ with bandwidth $\tau$. If both nodes are on the same processor, we have $c_{i, k}=0$.

In the second phase, we allocate the ready tasks to processors in the order given by their priority. We first compute the node with the highest priority, then the following node and so on. Let $P_{j}$ be a candidate processor for task $N_{i}$. We note $\operatorname{pct}\left(N_{i}, P_{j}\right)$ the partial completion time of task $N_{i}$ on processor $P_{j}$, and $d r\left(N_{i}\right)$ the instant where all the data needed to compute $N_{i}$ is available by $P_{j}$, i.e. the time at which the last data item required by $N_{i}$ to begin its execution is available by $P_{j} . N_{i}$ may be computed after the instant $d r\left(N_{i}\right)$ and as soon as the processor $P_{j}$ is available. Let $\operatorname{proc}\left(N_{k}\right)$ be the processor which executes task $N_{k}$. We deduce the following equations for $\operatorname{dr}\left(N_{i}\right)$ and $\operatorname{pct}\left(N_{i}, P_{j}\right)$ :

- For an entry task, we have:

$$
\operatorname{pct}\left(N_{i}, P_{j}\right)=e_{i, j}
$$

- For any other task, we have:

$$
\begin{aligned}
& \operatorname{pct}\left(N_{i}, P_{j}\right)=e_{i, j}+\max \left(\text { Available }[j]+d r\left(N_{i}\right)\right) \\
& d r\left(N_{i}\right)=\max _{N_{k} \in \operatorname{Pred}\left(N_{i}\right)}\left(c_{k, i}+\operatorname{pct}\left(N_{k}, \operatorname{proc}\left(N_{k}\right)\right)\right)
\end{aligned}
$$

where Available $[j]$ is the instant where processor $P_{j}$ is free to start the execution of a new task.

The task $N_{i}$ is mapped onto the processor which minimizes $\operatorname{pct}\left(N_{i}, *\right)$.

$$
\begin{aligned}
& \text { The PCT AlgORITHM } \\
& \text { 1: Compute the priority for each task } \\
& \text { 2: ReadyTask } \leftarrow \text { Entry tasks }\} \\
& \text { 3: While ReadyTask is not empty } \\
& \text { 4: Choose } n \text { in ReadyTask with the highest priority } \\
& \text { 5: Compute pct }(n, p) \text { for all } p \text { in } \mathcal{P} \\
& \text { 6: Assign } n \text { to the processor which minimize } p c t(n, p) \\
& \text { 7: } \quad \text { Update } A[p] \text { and ReadyTask } \\
& \text { 8: End while }
\end{aligned}
$$




\subsection{Best imaginary level (BIL) algorithm}

Oh and Ha present in [6] a list scheduler. The general idea is to assign a priority, or a static level, to each node. Then the list scheduler schedules the runnable nodes in the decreasing order of priority, and tries to determine the optimal processor for the selected node.

They define the level of a node $N_{i}$ as the Best Imaginary Level $(B I L)$. The BIL is the length of the critical path beginning with $N_{i}$ if this node is remapped onto a processor $P_{j}$, including the communications and assuming that all the children are perfectly scheduled. Since it is not always possible to schedule the nodes at the best times, we use the term imaginary:

$$
B I L\left(N_{i}, P_{j}\right)=e_{i, j}+\max _{N_{k} \in S u c c\left(N_{i}\right)}\left[\min \left(B I L\left(N_{k}, P_{j}\right), \min _{p \neq j}\left(B I L\left(N_{k}, P_{p}\right)+c_{i, p}\right)\right)\right]
$$

The $B I L$ of a node is then used to compute a priority order over the nodes.

Once the $B I L$ is computed for each node, we start the second phase which consists of selecting a node, i.e. computing a priority order. To select a node, we adjust the level of a $N_{i}$ on processor $P_{j}$ to measure the Best Imaginary Makespan $(B I M)$. BIM is defined as follows: $B I M\left(N_{i}, P_{j}\right)=$ Available $[j]+B I L\left(N_{i}, P_{j}\right)$. For each node, there exist $p$ different $B I M$ values, one for each processor. Assuming there exist $k$ runnable nodes at a step, we define the priority of a node $N_{i}$ as the $k^{t h}$ smallest $B I M$ value, or the largest finite $B I M$ value if the $k^{t h}$ is undefined. The selected node is the one with the highest priority.

Once we have selected a node, we have to find a processor where to map it. If the number of ready nodes $k$ is high, i.e. greater than the number of processors, the execution time becomes more important than the communication overhead, since the communication overhead is likely to be hidden. Therefore, we define the revised $B I M$ as follows :

$$
B I M^{*}\left(N_{i}, P_{j}\right)=B I M\left(N_{i}, P_{j}\right)+e_{i, j} \times \max \left(\frac{k}{P}-1,0\right)
$$

We select the processor that has the highest revised $B I M$ value. If more than one processor have the same revised $B I M$ value, we select the processor that makes the sum of the revised $B I M$ values of other nodes on the processor maximum. As soon as the task is assigned to a processor, we update the runnable nodes and continue while there exists a ready task.

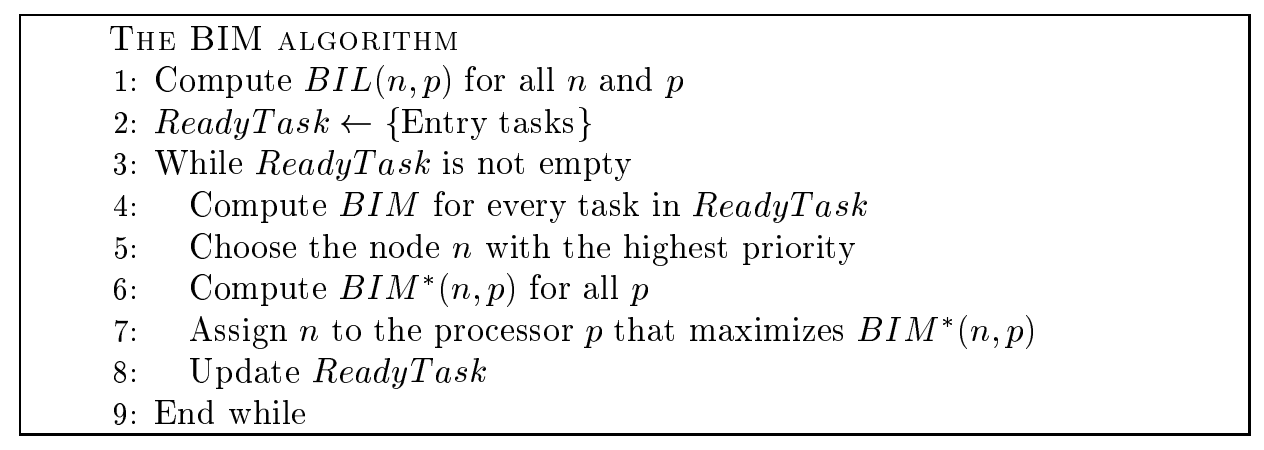

\subsection{Heterogeneous earliest finish time (HEFT) and critical path on a processor (CPOP) algorithms}

Topcuoglu, Hariri and Wu present in [9] two heuristics. The general idea of both heuristics is the same. In a first phase, they compute a priority on the runnable nodes and select the node with the highest priority. Then, in the second phase, using two different criteria, they determine a processor to which the selected node is mapped. Before studying the two different algorithms, we need some definitions. We define the earliest start time, EST, and the earliest finish time, EFT, of node $N_{i}$ on processor $P_{j}$ as follows :

$$
\begin{gathered}
\operatorname{EST}\left(N_{i}, P_{j}\right)=\max \left(A[j], \max _{N_{k} \in \operatorname{Pred}\left(N_{i}\right)}\left(\operatorname{EFT}\left(N_{k}, \operatorname{proc}\left(N_{k}\right)\right)+c_{k, i}\right)\right) \\
\operatorname{EFT}\left(N_{i}, P_{j}\right)=e_{i, j}+\operatorname{EST}\left(N_{i}, P_{j}\right)
\end{gathered}
$$


where $\operatorname{proc}\left(N_{k}\right)$ is the processor where $N_{k}$ is assigned. EST returns the ready time, i.e. the time when all data needed by $N_{i}$ is available at the host $P_{j}$, and when the host $P_{j}$ itself is available.

In the algorithm, tasks are ranked upward and downward to set the scheduling priorities. The upward rank of a task $N_{i}$ is recursively defined by

$$
\operatorname{rank}_{u}\left(N_{i}\right)=\overline{e_{i}}+\max _{N_{k} \in \operatorname{Succ}\left(N_{i}\right)}\left(c_{i, k}+\operatorname{rank}_{u}\left(N_{k}\right)\right)
$$

where $\bar{e}_{i}=\sum \frac{e_{i, j}}{p_{j}}$ is the average execution time of the task $N_{i}$ over the processors. rank $k_{u}$ is the length of the critical path from $N_{i}$ to the exit node, including the computation cost of the node itself. Similarly, the downward rank of a task $N_{i}$ is recursively defined by

$$
\operatorname{rank}_{d}\left(N_{i}\right)=\max _{N_{k} \in \operatorname{Pred}\left(N_{i}\right)}\left(c_{k, i}+\overline{e_{k}}+\operatorname{rank}_{d}\left(N_{k}\right)\right)
$$

The rank $_{d}$ is the largest distance from the start node to the node $N_{i}$, excluding the computation cost of the node itself.

\subsubsection{HEFT}

To set priority to a task $N_{i}$, the HEFT algorithm uses the upward rank value of the task. Ready tasks are sorted with respect to decreasing $r a n k_{u}$ values. If two nodes to be scheduled have the same priority, one of them is selected randomly.

The HEFT algorithm uses the EFT value to select the processor for the selected task. It is natural to consider the EFT value to select a processor. Indeed, when all nodes in the graph have been scheduled, the schedule length is the earliest finish time of the exit node. We assign node $N_{i}$ to the processor $P_{j}$ that minimizes the value of $\operatorname{EFT}\left(N_{i}, P_{j}\right)$.

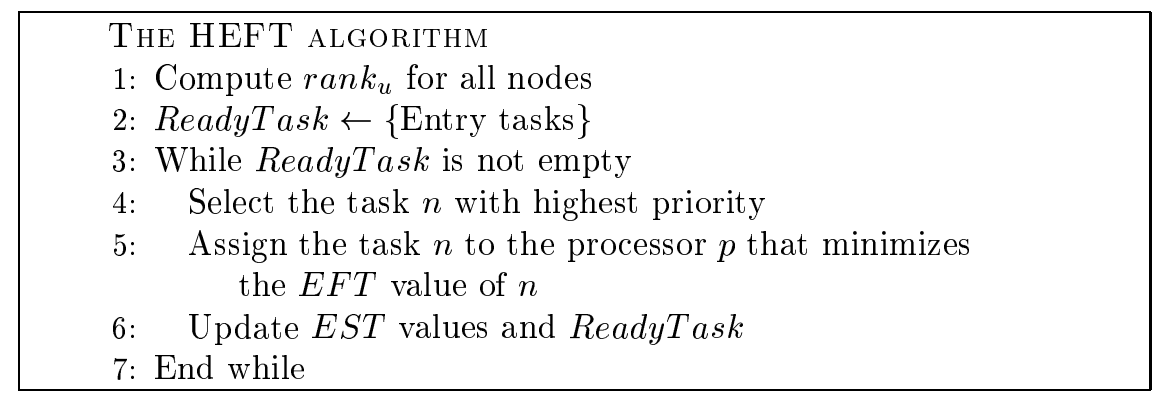

\subsubsection{CPOP}

The CPOP algorithm uses $\operatorname{rank}_{u}(n)+\operatorname{rank}_{d}(n)$ to assign the node priority. As previously, we select the node with the highest priority, i.e. we first consider the tasks that belong to the critical path. A task is on the critical path if its value of $r a n k_{u}+r a n k_{d}$ is equal to the value of $\operatorname{rank}_{u}\left(N_{s}\right)$ where $N_{s}$ is the start node. The critical-path-processor $(C P P)$ is the one that minimizes the length of the critical path. If the current task is on the critical path, then it is assigned to the $C P P$, otherwise it is assigned to the processor that minimizes the EFT. The time needed to compute the tasks along the critical path is a lower bound of the execution time. Hence it appears to be a good criterion in order to (try to) minimize the length of the critical path. The $C P P$ is often the fastest processor.

\subsection{Generalized dynamic level (GDL) algorithm}

Sih and Lee propose in [8] a compile-time scheduling heuristic for heterogeneous networks called $G D L$. As in the previous algorithm, the $G D L$ scheduler computes the critical path in a heterogeneous system. Contrarily to the $C P O P$ algorithm, $G D L$ defines the assumed execution time of node $N_{i}$ denoted $e^{*}\left(N_{i}\right)$ as the median execution time of the node over all the processors. 


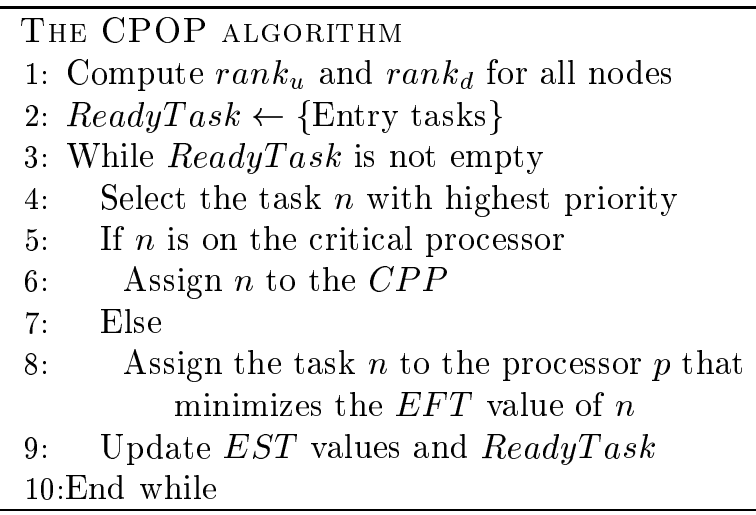

We define the static level of a node $N_{i}, S L\left(N_{i}\right)$ as the largest sum of execution times along any directed path from $N_{i}$ to an exit node of the graph. $S L\left(N_{i}\right)$ can be easily computed recursively. To take the difference in processor speeds into account, we introduce

$$
\Delta\left(N_{i}, P_{j}\right)=e^{*}\left(N_{i}\right)-e_{i, j}
$$

Then, we introduce a dynamic level $D L\left(N_{i}, P_{j}\right)$ which reflects how well node $N_{i}$ and processor $P_{j}$ are matched. This quantity will be re-evaluated at each step of the algorithm to take next decisions into account and is given by

$$
D L\left(N_{i}, P_{j}\right)=S L\left(N_{i}\right)-E S T\left(N_{i}, P_{j}\right)+\Delta\left(N_{i}, P_{j}\right) .
$$

The term $\operatorname{EST}\left(N_{i}, P_{j}\right)$ is the earliest start time defined in the same way as for the HEFT and the CPOP algorithms. This algorithm is very simple. While there exists a ready task, we select the node and the processor which maximize the expression $D L$.

Descendant consideration Although $D L\left(N_{i}, P_{j}\right)$ indicates how well node $N_{i}$ is matched with processor $P_{j}$, it fails to consider how well the descendants of $N_{i}$ are matched with $P_{j}$. For each node $N_{i}$ we note $D\left(N_{i}\right)$ the descendant to which $N_{i}$ passes the most data and $d\left(N_{i}, D\left(N_{i}\right)\right)$ the amount of data passed between them. We then define $F\left(N_{i}, D\left(N_{i}\right), P_{j}\right)$ to indicate how quickly $D\left(N_{i}\right)$ can be completed on any other processor if $N_{i}$ is executed on $P_{j}$.

$$
F\left(N_{i}, D\left(N_{i}\right), P_{j}\right)=\tau \times d\left(N_{i}, D\left(N_{i}\right)\right)+\min _{k \neq j} E\left(D\left(N_{i}\right), P_{k}\right)
$$

This is a lower bound of the time necessary to finish the execution of $D\left(N_{i}\right)$ on any processor other than $P_{j}$. We then define a descendant consideration term as

$$
D C\left(N_{i}, P_{j}\right)=e^{*}\left(D\left(N_{i}\right)\right)-\min \left\{E\left(D\left(N_{i}\right), P_{j}\right), F\left(N_{i}, D\left(N_{i}\right), P_{j}\right)\right\}
$$

Resource scarcity We generally fail to consider how important it is for two nodes to obtain the same processor. To characterize this resource scarcity cost, we first define the preferred processor of a node, i.e. the processor that maximizes its dynamic level. We then define the cost of not scheduling $N_{i}$ onto its preferred processor by

$$
C\left(N_{i}\right)=D L\left(N_{i}, P_{j}^{*}\right)-\max _{k \neq j^{*}} D L\left(N_{i}, P_{k}\right)
$$

where $j^{*}$ is the index of the preferred processor of $N_{i}$. If the cost is zero, then there will be at least one processor leading to the same dynamic level for $N_{i}$

Generalized dynamic level By taking into account the descendant consideration and the resource scarcity we can now define a generalized dynamic level :

$$
G D L\left(N_{i}, P_{j}\right)=D L\left(N_{i}, P_{j}\right)+D C\left(N_{i}, P_{j}\right)+C\left(N_{i}\right)
$$

The algorithm is unchanged. We select among the runnable tasks the task and the processor which maximize the $G D L$. 


\begin{tabular}{l} 
THE GDL ALGORITHM \\
1: Compute $e^{*}(n)$ and $D(n)$ for all nodes $n$ \\
2: Compute $S L(n)$ for all nodes $n$ \\
3: ReadyTask $\leftarrow$ Entry tasks $\}$ \\
4: While ReadyTask is not empty \\
5: Compute GDL $(n, p)$ for every node $n$ in ReadyTask \\
6: $\quad$ and every processor $p$ \\
7: $\quad$ Update Reair $(n, p)$ that maximizes $G D L$ \\
8: End while \\
\hline
\end{tabular}

\section{The Iso-Level Heterogeneous Allocation (ILHA) algorithm}

The main idea of the algorithm that we propose is to perfectly balance the load between those computations that can be performed in parallel. First we sketch a simple method to balance a set of $n$ tasks on $p$ heterogeneous processors, then we explain how to split the task graph into task levels which we assign to processors using the load-balancing algorithm. Finally we outline the ILHA algorithm.

Load balancing algorithm Given $n$ independent chunks of computations, each requiring the same amount of work, how can we assign these chunks to $p$ processors so that the load is best balanced? Intuitively, the load of $P_{i}$ should be inversely proportional to its cycle-time $t_{i}$, i.e. $P_{i}$ should receive $c_{i}=\frac{\frac{1}{t_{i}}}{\sum_{j=1}^{p} \frac{1}{t_{j}}} \times n$ chunks. This strategy leads to a perfect load balance when $n$ is a multiple of $C=l c m\left(t_{1}, t_{2}, \cdots, t_{p}\right) \sum_{j=1}^{p} \frac{1}{t_{j}}$, a quantity that may be very large. For the general case, the following algorithm provides the best solution [2]:

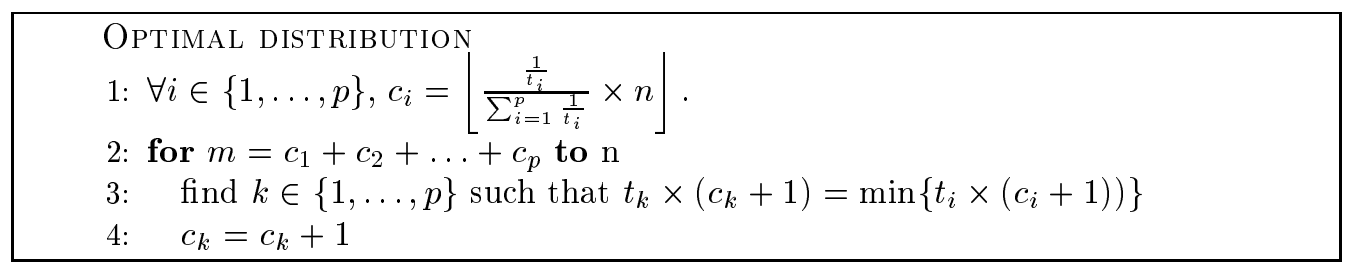

\subsection{First version}

Iso-level splitting We split a task graph into levels made up of independent tasks, by considering the tasks that will be ready at the same time-step. In other words, two tasks belong to the same level if they have the same top-level, using the terminology of [10]. This is done by a traversal of the graph. Initially, the 0 -level is composed of the entry tasks. The $(i+1)$-th level groups the tasks that are ready when the $i$-th level is achieved.

The IHLA-0 algorithm A first version of the $I L H A$ algorithm is the following: we traverse the task graph to split it into levels made of independent tasks. We compute the number of tasks that we allocate to each processor using the load-balancing algorithm. Once this is done, we have to determine exactly which task is given to each processor. The criteria is to minimize the communication costs. So for each task of the level, we consider its predecessors. If they are all allocated to the same processor, we try to allocate the task to the same processor (i.e. if the processor may receive another task), otherwise, we allocate the task to the fastest processor that is not yet saturated (able to receive new tasks according to the load-balancing strategy). This simple strategy leads to the following algorithm:

\subsection{Refined version}

In the previous version of the ILHA algorithm, we process all the ready tasks at each step. In some cases, it would be better to take into account the bottom level of the ready tasks and to consider first the tasks on a 


The ILHA ALGORITHM
1: ReadyTask $\leftarrow$ Entry tasks $\}$
2: While ReadyTask is not empty
3: $\quad$ Compute the optimal distribution with $\|$ ReadyTask $\|$ tasks
4: $\quad$ For each task $t$ of ReadyTask
5: $\quad$ If all predecessors of $t$ are on $p$ and $p$ is free
6: Assign $t$ to $p$
7: $\quad$ For each task $t$ of ReadyTask not yet assigned
8: Assign $t$ to the first free processor
9: Update ReadyTask
10:End while

critical path. To this purpose, we sort the ready tasks according to their bottom level. Then, we introduce a parameter $B$, the maximal number of ready tasks that will be considered at each step. We consider those $B$ tasks with the higher bottom levels and we allocate them using the load balancing algorithm. Then, we update the set of ready tasks (indeed some new tasks may have become ready) and we re-sort them according to their bottom level. Thus, we expect that the tasks on a critical path will be processed as soon as possible. Unfortunately, we face a tradeoff for choosing an appropriate value for $B$. On one hand if $B$ is large, it will be possible to better balance the load and minimize the communications. On the other hand, a small value of $B$ will enable us to process the tasks on the critical path sooner. Of course $B$ must be at least equal to the number of processors, otherwise some processors will be kept idle. The choice of $B$ will be discussed furthermore in the Section 5 .

We obtain the final version of the $I L H A$ algorithm:

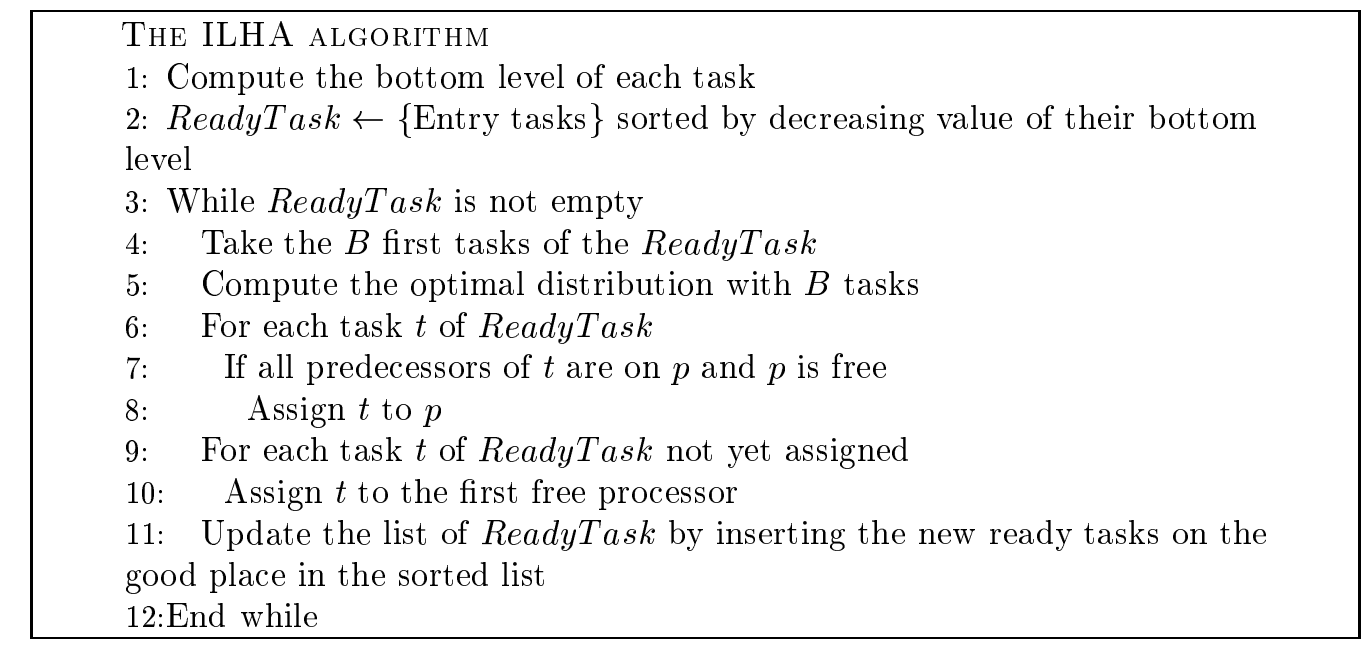

\section{$5 \quad$ Experiments}

\section{$5.1 \quad$ Testbeds}

In order to compare the different algorithms, we consider six classical kernels representing various types of parallel algorithms. The selected task graphs are:

- LU: LU decomposition

- LAPLACE: Laplace equation solver

- STENCIL: stencil algorithm 
- FORK-JOIN: fork-join graph

- DOOLITTLE: Doolittle reduction

- LDMt: $L D M^{t}$ decomposition

Miniature versions of each task graph are shown in Figure 1.

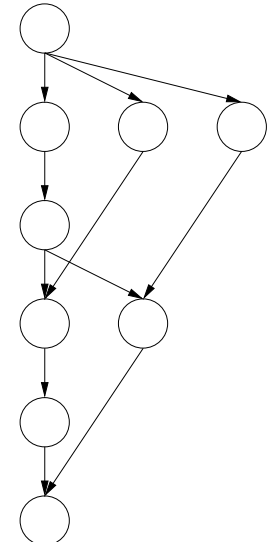

The LU task graph

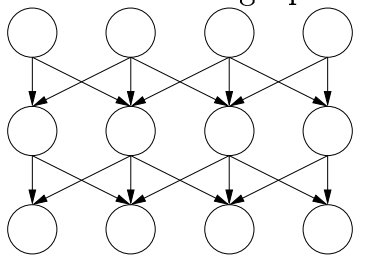

The stencil task graph

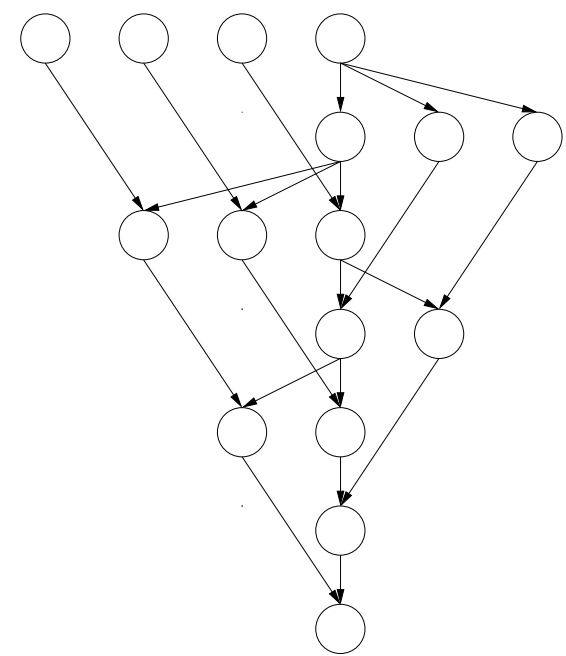

The DOOLITTLE reduction task graph

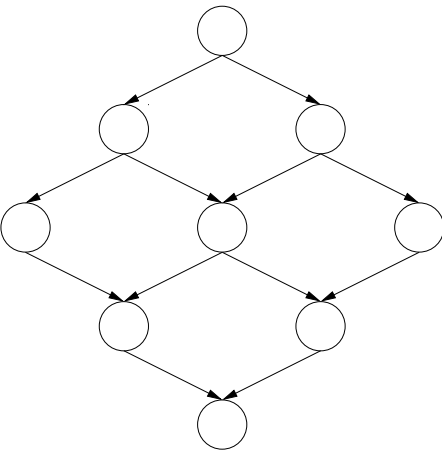

The LAPLACE task graph

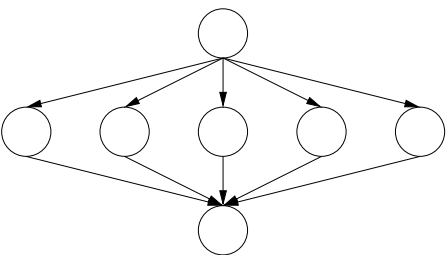

The fork-join task graph

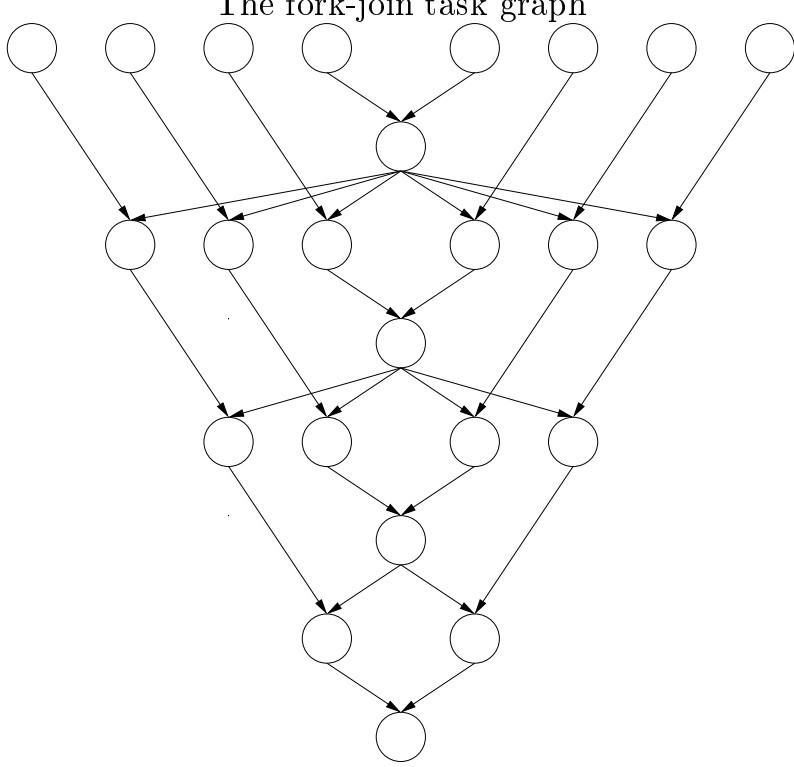

The $L D M^{t}$ decomposition task graph

Figure 1: The different task graphs

\subsection{Weights and speeds}

Task weights For the LAPLACE, STENCIL, and FORK-JOIN testbeds, all tasks have same weight, which we normalize to 1 . For the linear algebra testbeds, i.e. LU, DOOLITTLE and LDMt, the situation 
is more complicated, because the amount of work to be done at each step of the algorithm is not constant (see $[4,7]$ ). For the LU kernel, the weight of a task at level $k$ is $N-k$, where $N$ is the size of the graph. For the DOOLITTLE and LDMt kernels, the the weight of a task at level $k$ is $k$, where $k$ varies from 1 to $N$, the size of the graph.

Processor speeds We consider two sets of experiments. In the first set, we use only 3 processors with respective cycle times 6,10 and 15 . Remember that the time to execute a task is the product of its weight by the processor cycle-time. In the second set, we use 10 processors: five processors with cycle time 6 , three processors with cycle time 10, and two processors with cycle time 15.

Communication costs For each testbed, we let the communication costs be proportional to the task weights: indeed in each kernel, we always communicate the data that has just been updated. In other words, the communication cost from a task $v$ to a task $v^{\prime}$ is equal to $c$ times the weight of $v$, where $c$ is a parameter that models the communication-to-computation ratio of the target platform. For each kernel, we perform a first simulation with small communication costs, i.e. $c=1$, as well as a second simulation with large communication costs, i.e. $c=10$.

\subsection{Results}

In the first subset of experiments, we use only 3 processors with respective cycle times 6,10 and 15 . These three processor speeds are strongly heterogeneous: it turns out that many heuristics fail to be more efficient than a sequential execution. This should not be too surprising: if we were able to achieve a full utilization of the computing resources, i.e. an efficiency equal to one, we would improve the sequential time (using the fastest processor alone) by a factor $6 *\left(\frac{1}{6}+\frac{1}{10}+\frac{1}{15}\right)=2$ (which represents the maximum number of tasks that we may compute during a cycle time). In the second subset of experiments, we use a larger number of processors, namely 10 processors: 5 processors of cycle time 6 , three processors of cycle time 10 and two processors of cycle-time 15. This time, if we were able to achieve a full utilization of the computing resources, i.e. an efficiency equal to one, we would improve the sequential time (using one of the fastest processors alone) by a factor $\frac{38}{5}=7.6$.

\subsubsection{Results with three processors}

In Figures 2 to 13, we show the expected execution time of the five heuristics (BIL, GDL, CPOP, HEFT and PCT) and of two instances of the ILHA heuristic with different values for $B$, namely $B=10$ and $B=20$.

For the LAPLACE problem (Figures 2 and 3), BIL, HEFT and the two versions of ILHA have very good results. As they perfectly balance the load and overlap communications and computations, there is no difference between the experiments with the two different values of the communication cost. GDL and CPOP are equivalent to a sequential scheduling in both cases. Finally, PCT presents very poor results, worse than a sequential execution (and these poor results are even worse when the communication cost increases).

For the STENCIL problem (Figures 4 and 5), contrarily to the LAPLACE problem, only HEFT and ILHA present good results. The remarks concerning these two heuristics remains valid. One more time, GDL and CPOP amount to a sequential scheduling, because they assign all the tasks to the fastest processor. PCT presents bad results again. The more interesting fact is that, contrarily to the LAPLACE problem, BIL presents poor results: in the STENCIL problem, there is a lot of potential communications, and BIL generates too many of them to be efficient.

For the LU problem (Figures 6 and 7), HEFT is the best scheduler. This time, CPOP and PCT present similar results as HEFT. These three algorithms are slightly better than the two versions of ILHA. The important fact is that we see here the interest to decrease the value of $B$. Indeed, the ILHA version with $B=10$ is quite better than the ILHA version with $B=20$. The difference between the two versions is more visible when the communication cost increases. It is the only impact of the communication cost in these experiments. BIL and GDL have poor results: their expected execution times are twice greater than those of the other algorithms.

For the FORK-JOIN problem (Figures 8 and 9), GDL and CPOP find a sequential scheduling. The other heuristics (BIL, HEFT, PCT and ILHA) obtain very good results and are optimal. Indeed, they achieve 


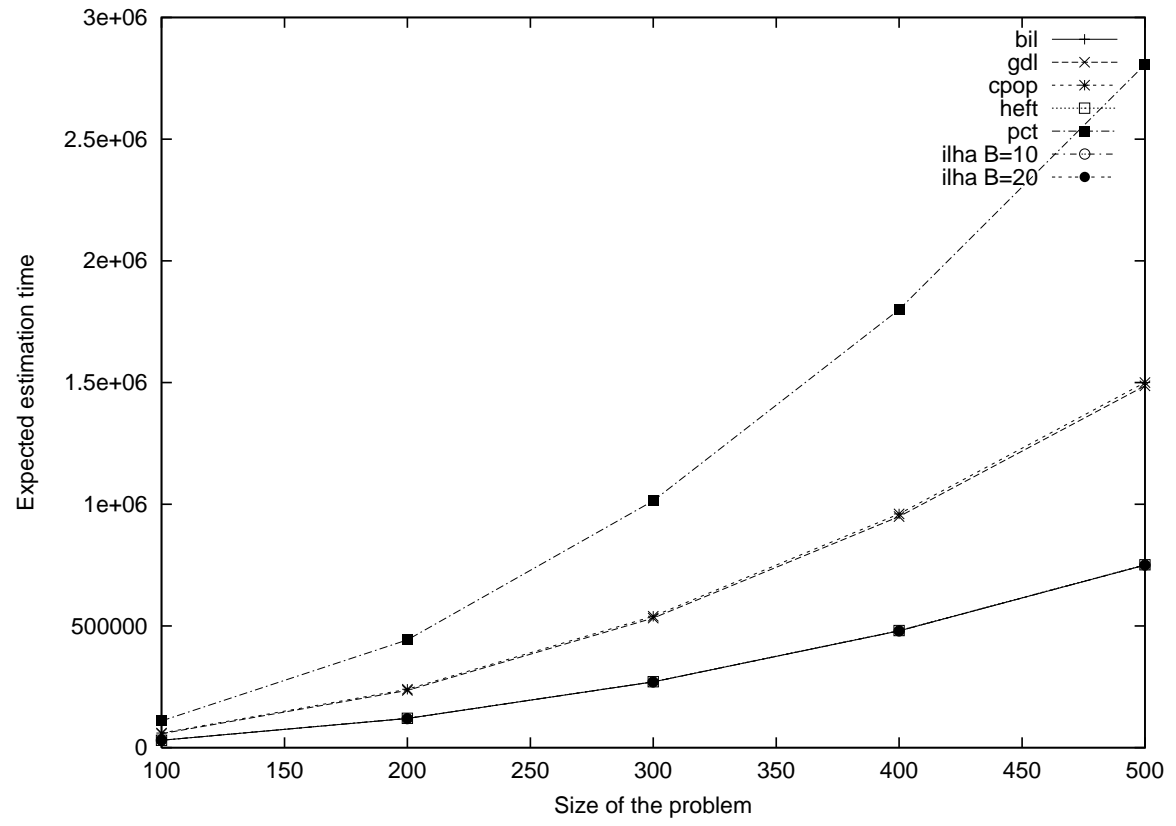

Figure 2: Comparison of the different heuristics for the LAPLACE problem, with 3 processors of cycle-time 6,10 and 15 and a communication cost equal to 1.

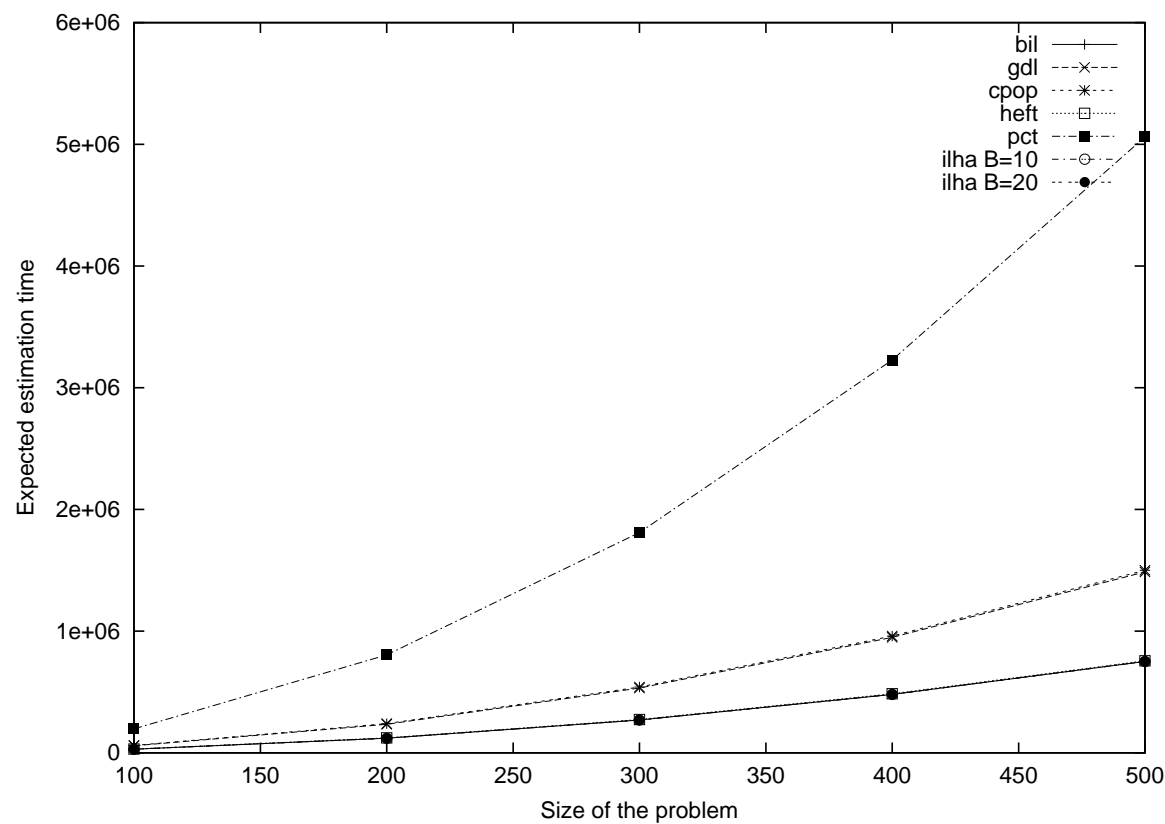

Figure 3: Comparison of the different heuristics for the LAPLACE problem, with 3 processors of cycle-time 6, 10 and 15 and a communication cost equal to 10. 


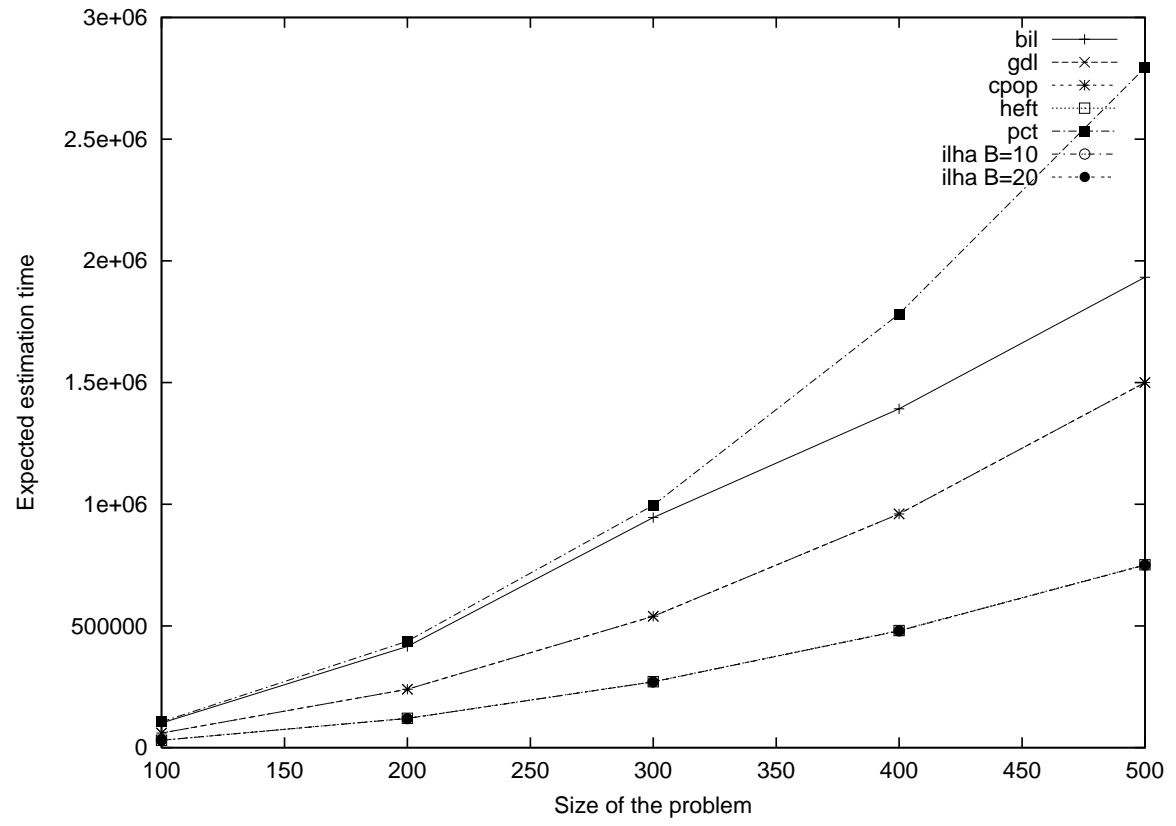

Figure 4: Comparison of the different heuristics for the STENCIL problem, with 3 processors of cycle-time 6,10 and 15 and a communication cost equal to 1.

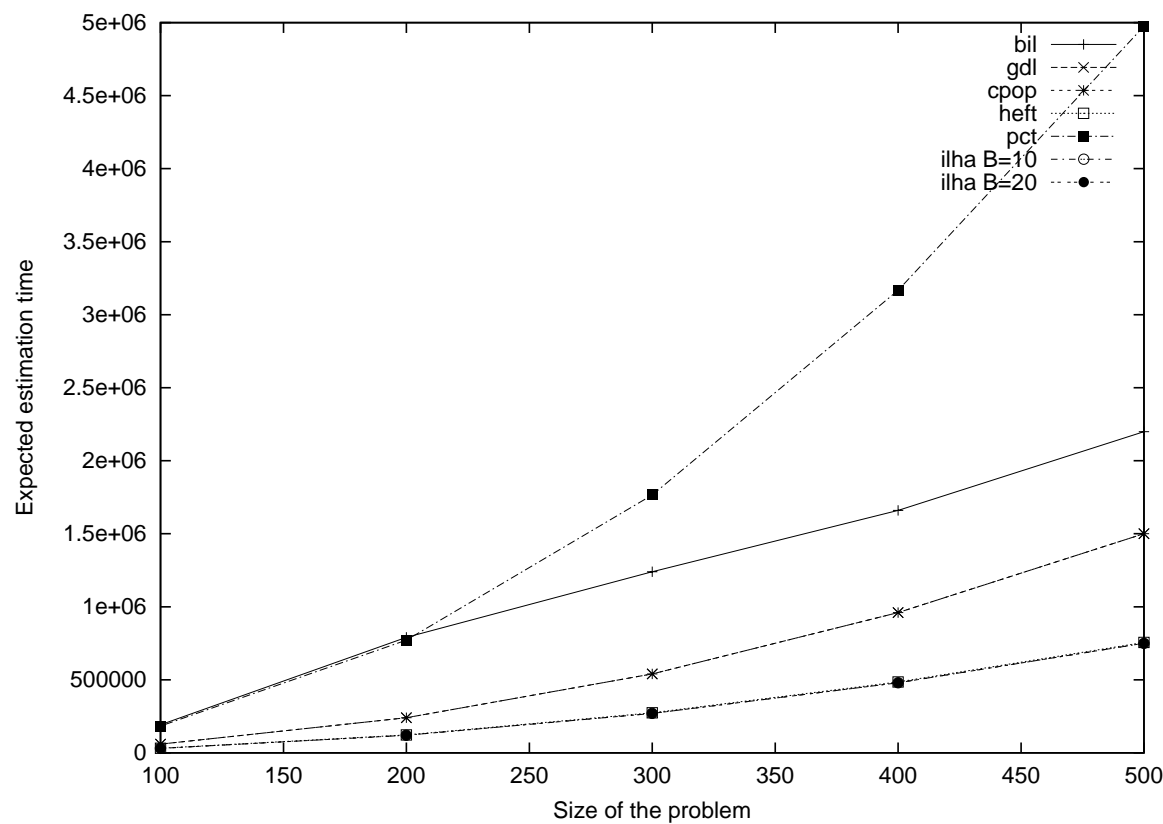

Figure 5: Comparison of the different heuristics for the STENCIL problem, with 3 processors of cycle-time 6, 10 and 15 and a communication cost equal to 10. 


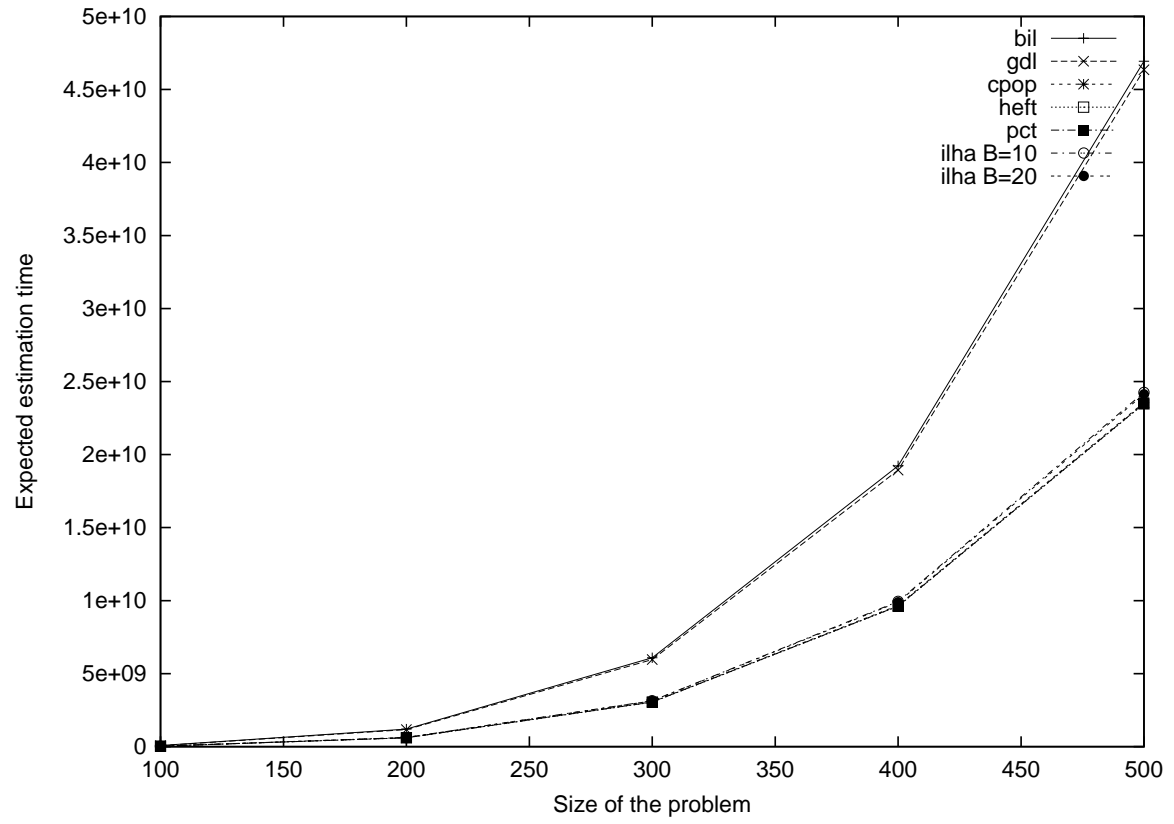

Figure 6: Comparison of the different heuristics for the LU problem, with 3 processors of cycle-time 6, 10 and 15 and a communication cost equal to 1.

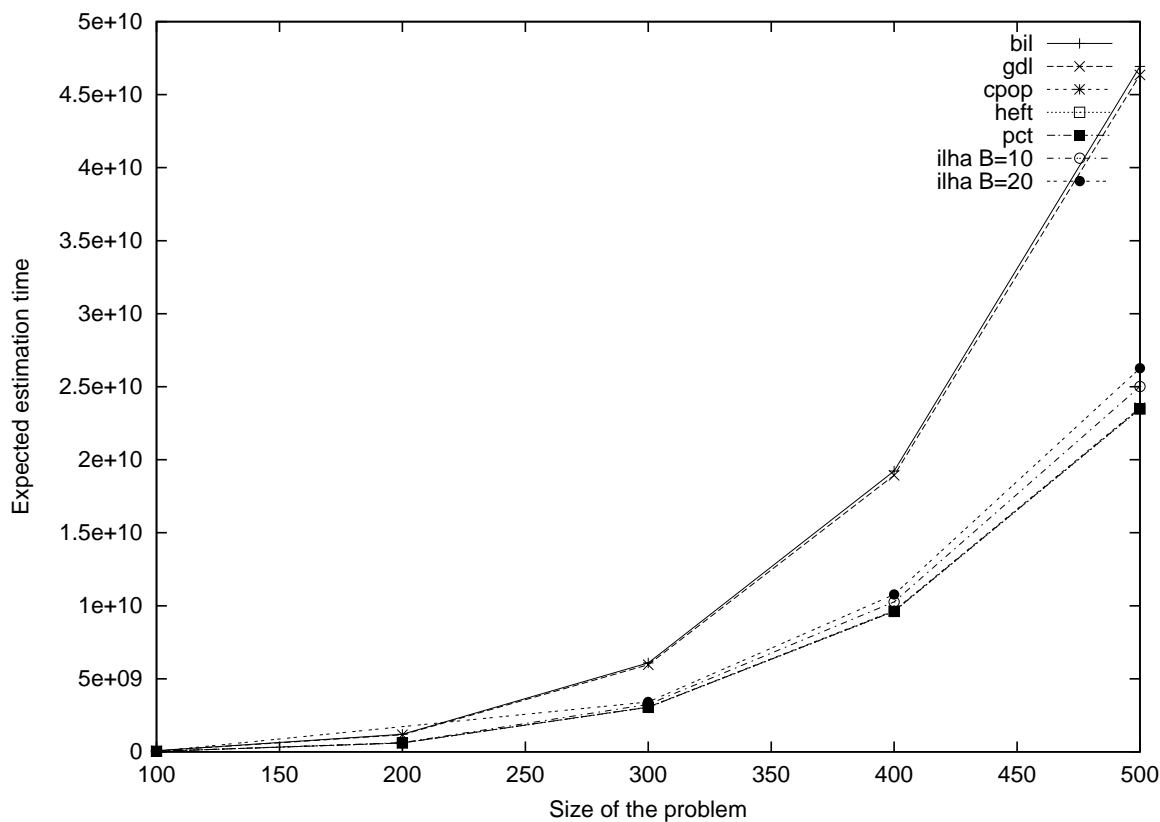

Figure 7: Comparison of the different heuristics for the LU problem, with 3 processors of cycle-time 6,10 and 15 and a communication cost equal to 10. 


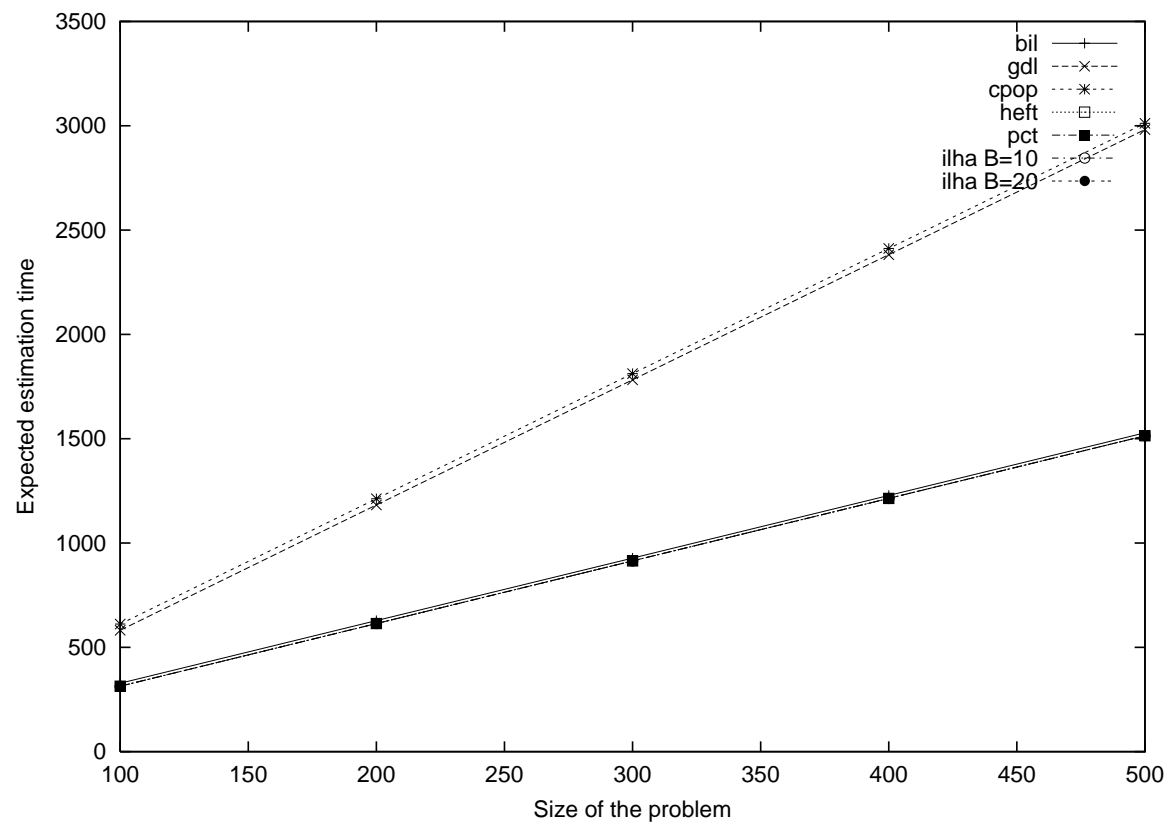

Figure 8: Comparison of the different heuristics for the FORK-JOIN problem, with 3 processors of cycle-time 6,10 and 15 and a communication cost equal to 1.

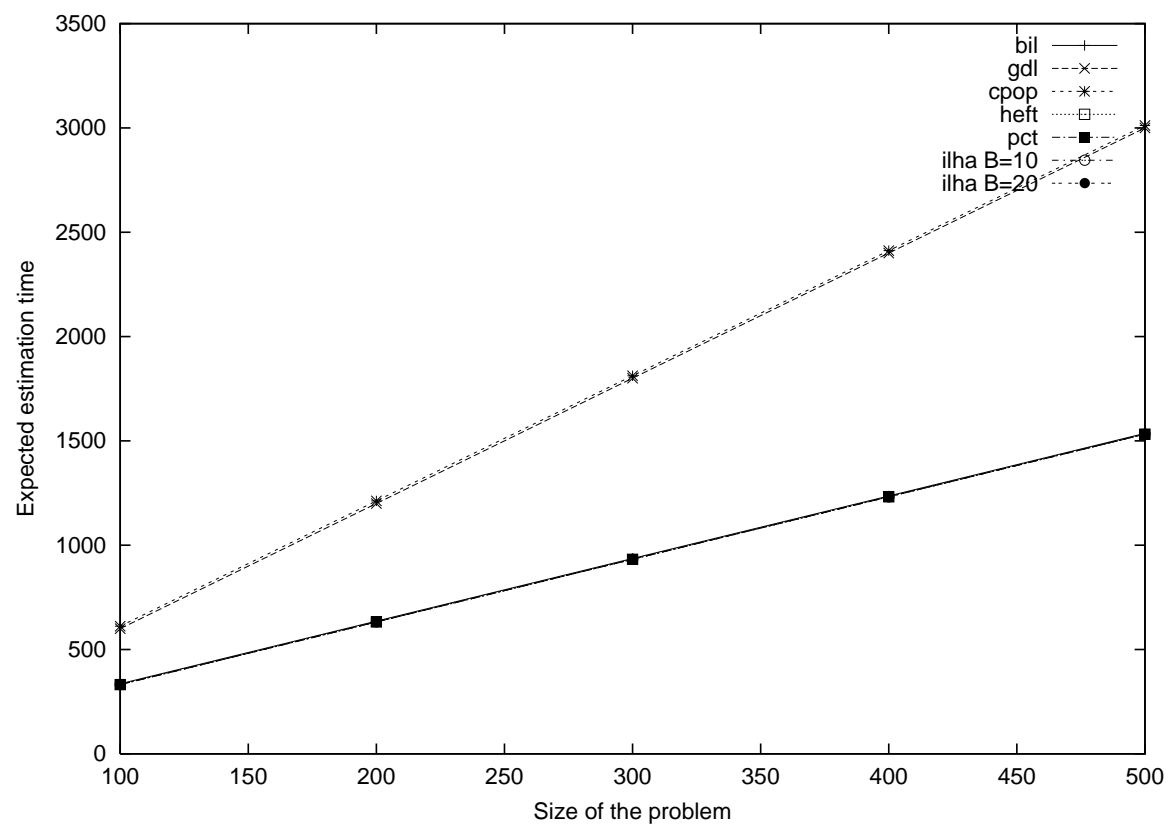

Figure 9: Comparison of the different heuristics for the FORK-JOIN problem, with 3 processors of cycle-time 6, 10 and 15 and a communication cost equal to 10. 
a full utilization of the resources and again a factor of 2 over the sequential time. The influence of the communication cost is very low, because all the communications are overlapped by the computations.

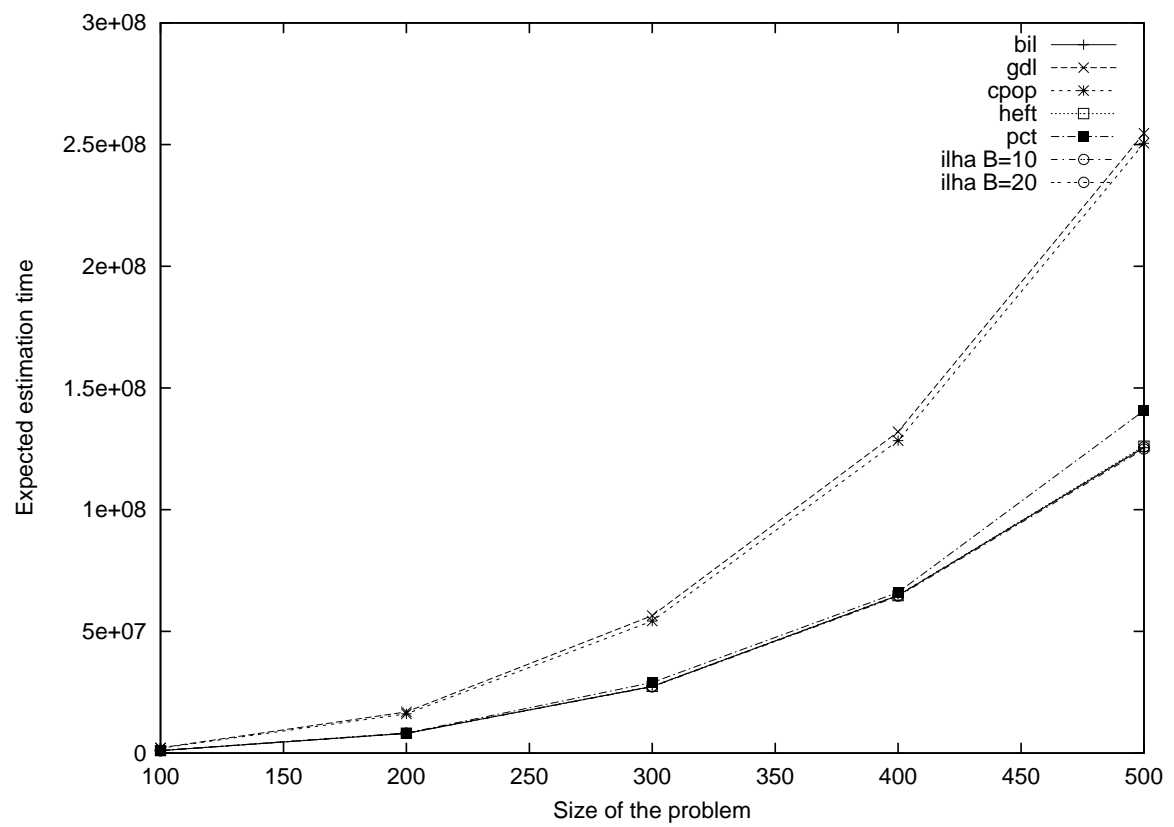

Figure 10: Comparison of the different heuristics for the DOOLITTLE problem, with 3 processors of cycletime 6,10 and 15 and a communication cost equal to 1.

For the DOOLITTLE problem (Figures 10 and 11), the best results are obtained with HEFT, ILHA and BIL. PCT is close to the three previous algorithms. The expected execution times of GDL and CPOP are slighlty better than the sequential time. The ratio between HEFT-ILHA-BIL and GDL-CPOP is close to 2. HEFT, ILHA and BIL are close to the optimal solution. In this case, as for the LU problem, the communication cost has no effect, but the value of $B$ influences the results of the ILHA algorithm, even though these effects are less important here.

For the LDMt problem (Figures 12 and 13), the conclusions are exactly the same as for the DOOLITTLE problem.

\subsubsection{Results with 10 processors}

In Figures 14 to 25, we show the expected execution time of the five heuristics (BIL, GDL, CPOP, HEFT and PCT) and of the ILHA heuristic with $B=38$. With this value of $B$, we are able to perfectly balance the load of the processors. Indeed we give 5 tasks to each processor of cycle time 6 (hence 30 tasks), 3 tasks to each processor of cycle time 10 (hence 9 tasks) and finally 2 tasks to each processor of cycle time 15 (hence 4 tasks). So in 30 time-units we process $25+9+4=38$ tasks. To compute these 38 tasks in a sequential way, using one of the fastest processos, we need $38 \times 6=228$ time-units. So we may improve the sequential time by a factor at most $\frac{228}{30}=7.6$.

For the LAPLACE problem (Figures 14 and 15), the conclusions obtained with 3 processors remain valid. However, the differences between all the algorithms are increased. The solution given by CPOP is still equivalent to the sequential scheduling. GDL is a little better than the sequential scheduling. PCT shows bad results which become even worse when the communication cost increases. The main interesting fact is that we can see in these figures that HEFT, BIL and ILHA asymptotically reach a speedup of 7.6. These three algorithms use three different schedules but they all perfectly balance the load over all the processors.

For the STENCIL problem (Figures 16 and 17), we note that ILHA and HEFT perfectly balance the load and achieve a full utilization of the resources. We can measure a speedup close to 7.6 over the sequential time achieved by CPOP and GDL. BIL and PCT present bad results. Note that that the communication cost has a small effect on BIL while it strongly affects PCT. 


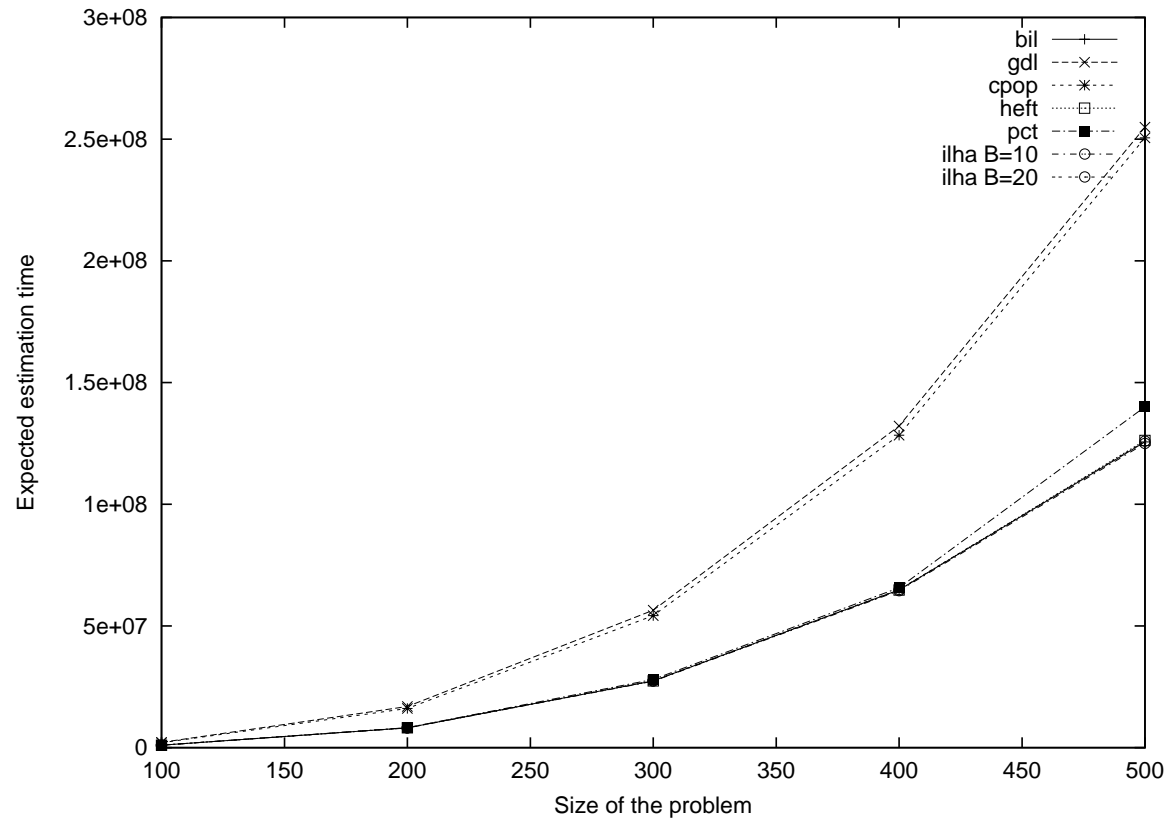

Figure 11: Comparison of the different heuristics for the DOOLITTLE problem, with 3 processors of cycletime 6,10 and 15 and a communication cost equal to 10 .

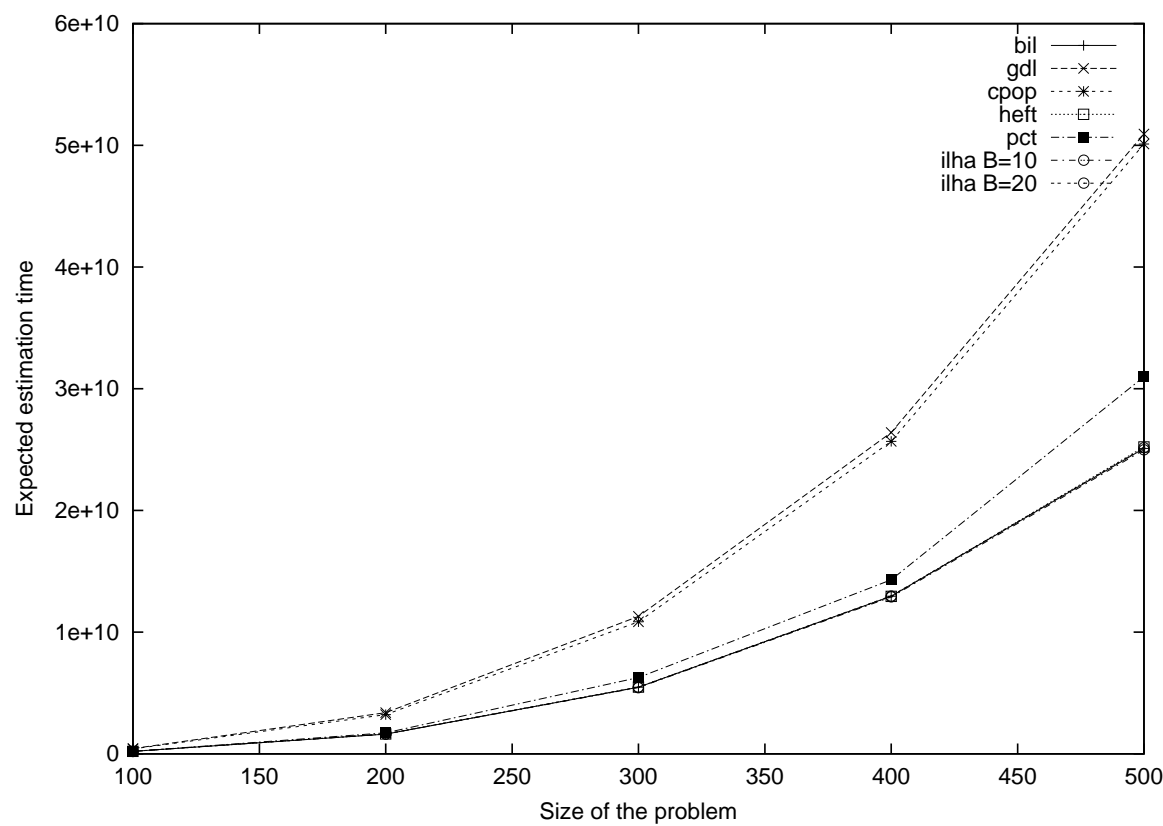

Figure 12: Comparison of the different heuristics for the LDMt problem, with 3 processors of cycle-time 6 , 10 and 15 and a communication cost equal to 1. 


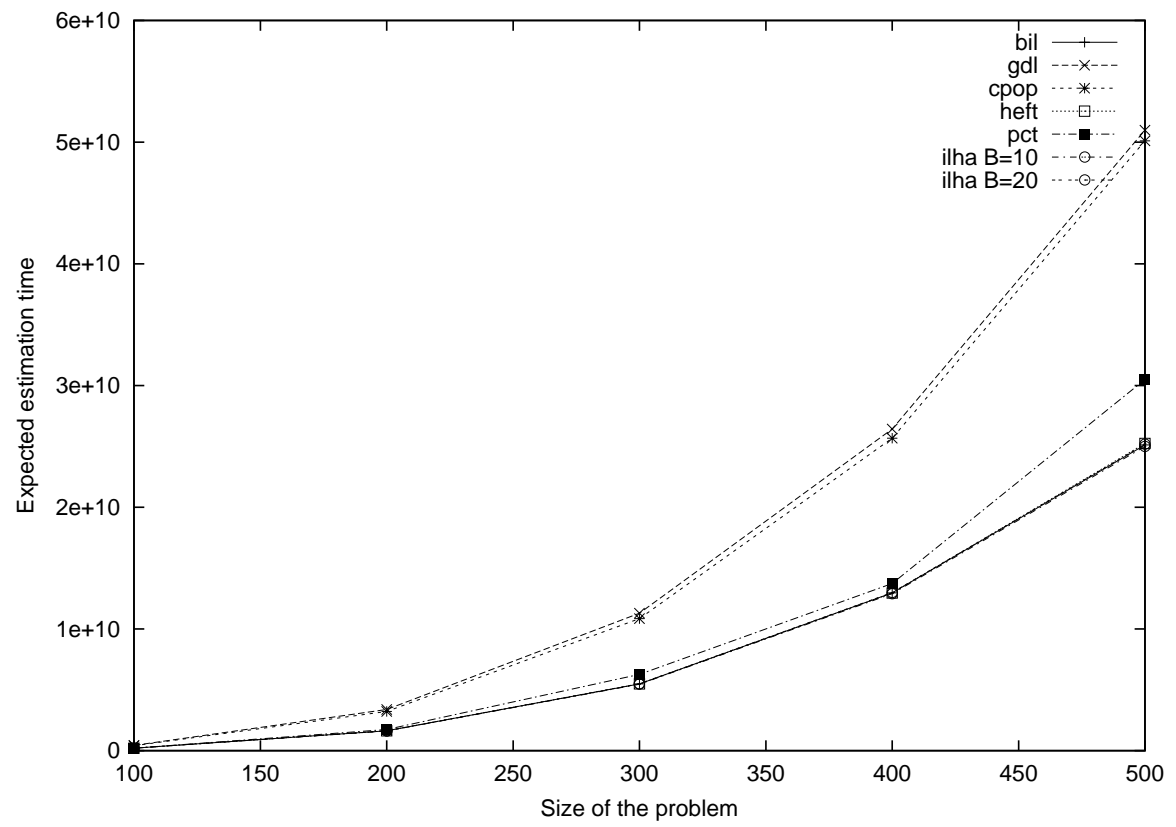

Figure 13: Comparison of the different heuristics for the LDMt problem, with 3 processors of cycle-time 6 , 10 and 15 and a communication cost equal to 10 .

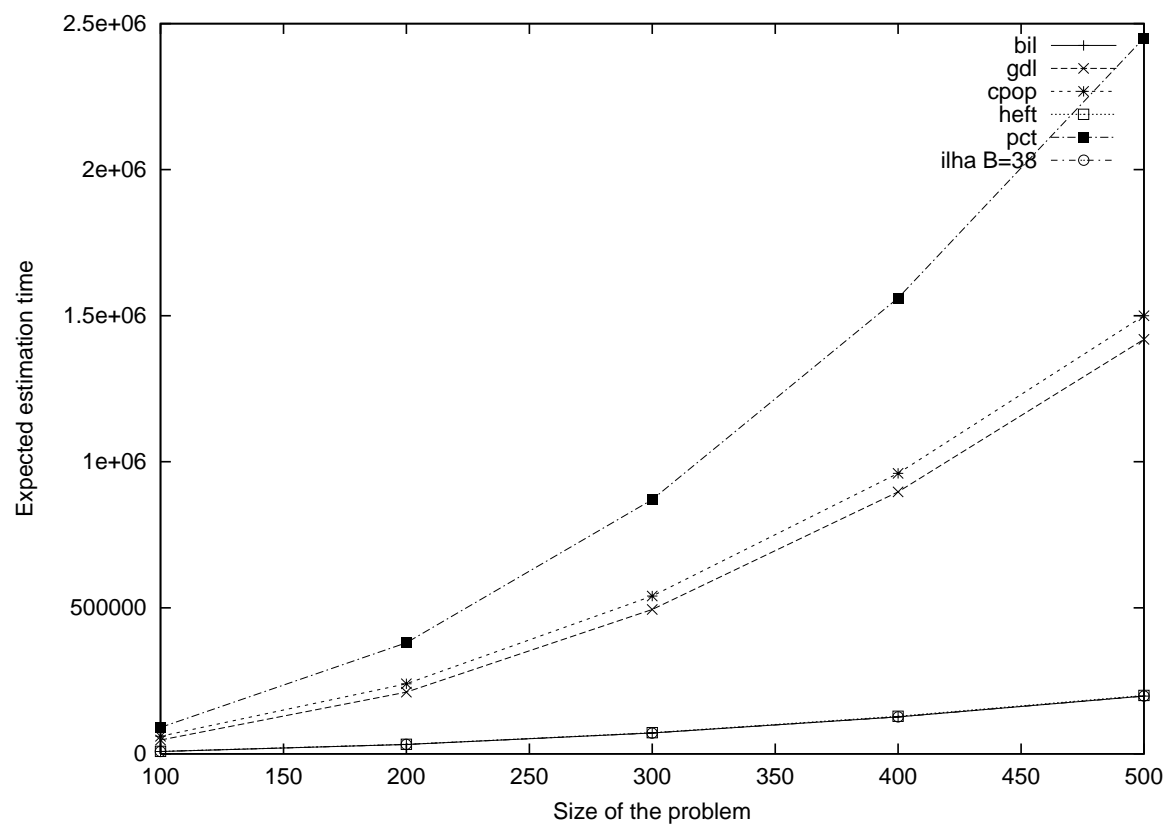

Figure 14: Comparison of the different heuristics for the LAPLACE problem, with 10 processors and a communication cost equal to 1. 


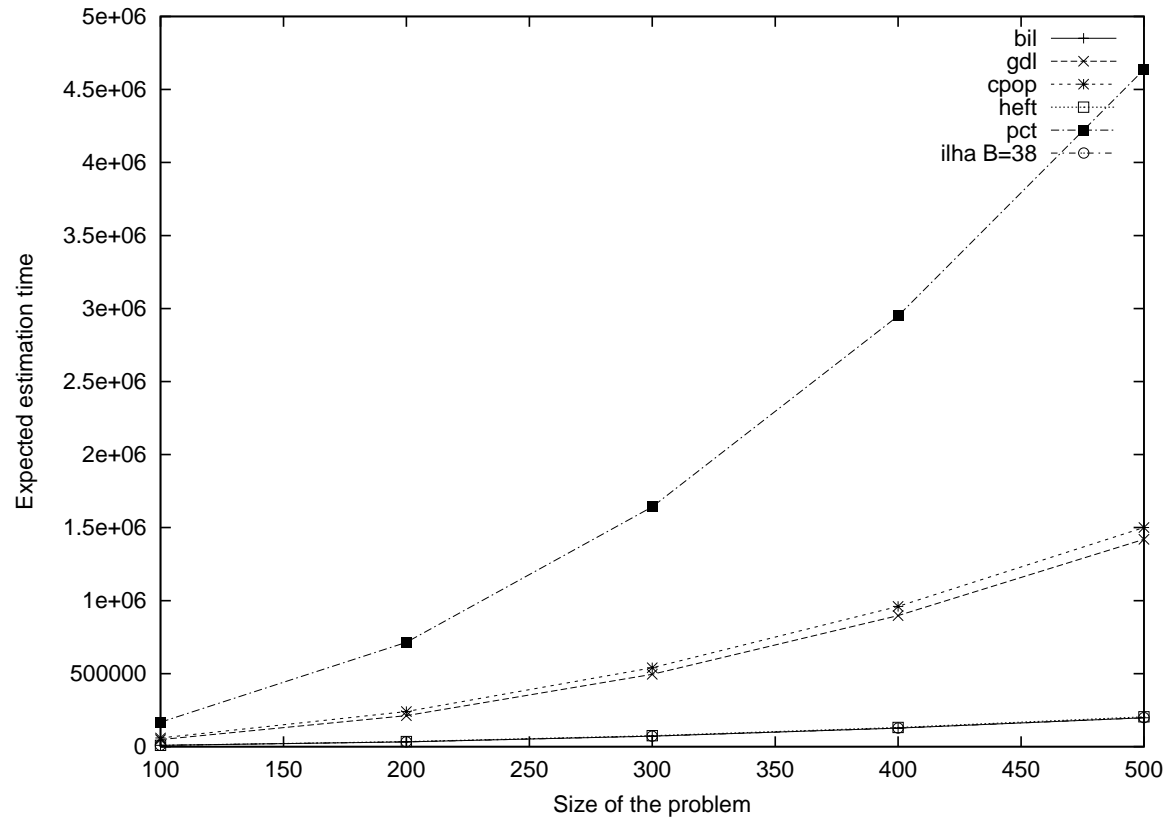

Figure 15: Comparison of the different heuristics for the LAPLACE problem, with 10 processors and a communication cost equal to 10 .

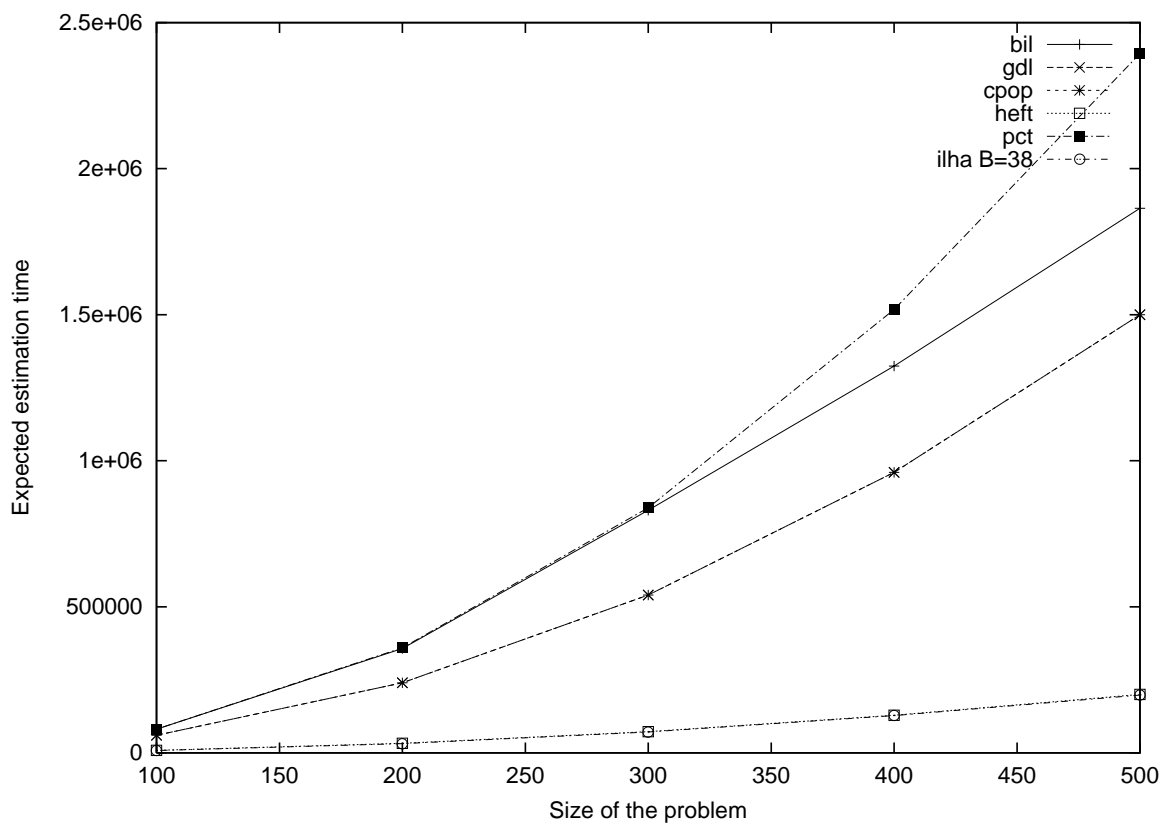

Figure 16: Comparison of the different heuristics for the STENCIL problem, with 10 processors and a communication cost equal to 1 . 


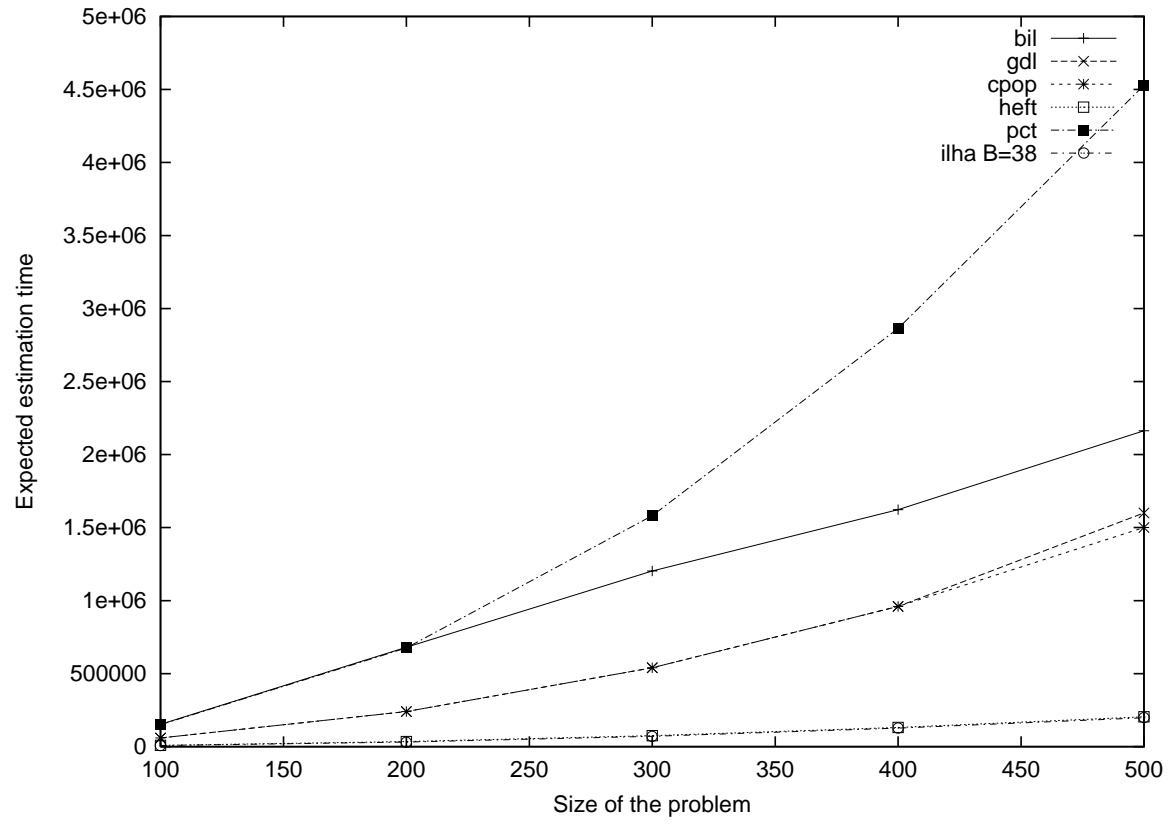

Figure 17: Comparison of the different heuristics for the STENCIL problem, with 10 processors and a communication cost equal to 10 .

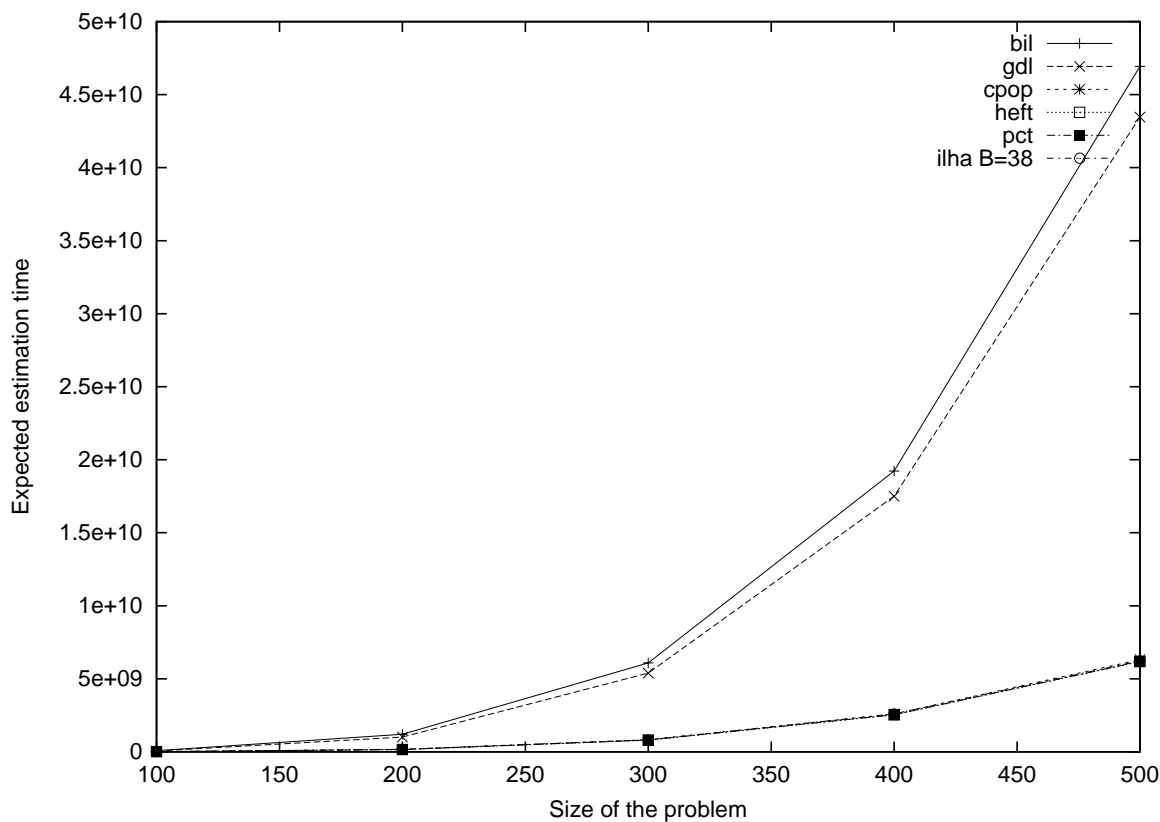

Figure 18: Comparison of the different heuristics for the LU problem, with 10 processors and a communication cost equal to 1. 


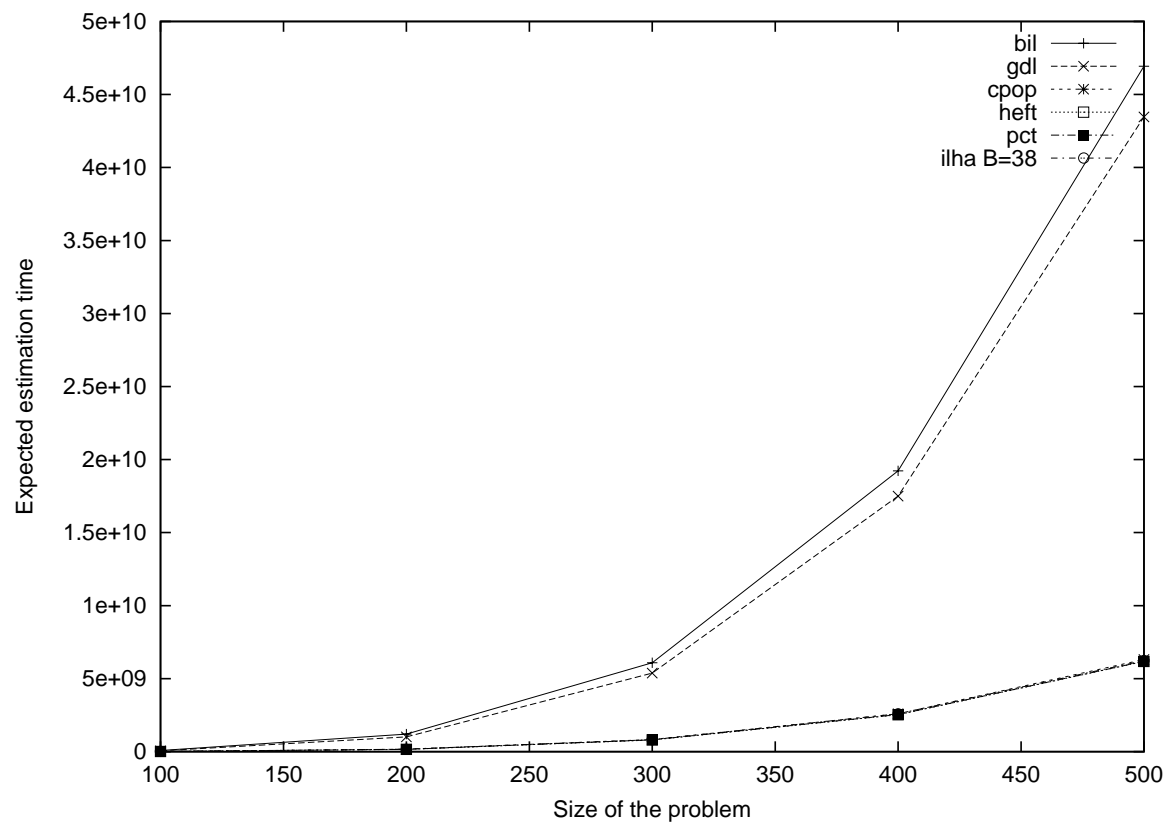

Figure 19: Comparison of the different heuristics for the LU problem, with 10 processors and a communication cost equal to 10 .

For the LU problem (Figures 18 and 19), BIL and GDL are close to the sequential time when the four other algorithms present very good results. The ratio between these two sets of curves is around 7.5. It seems that HEFT, CPOP, PCT and ILHA perfectly balance the load over the processors and so achieve a full utilization of the resources.

For the FORK-JOIN problem (Figures 20 and 21), HEFT, ILHA and PCT have very good results and asymptotically reach the theoretical speedup. CPOP gives the sequential schedule for the fork-join problem. GDL is slightly better than the sequential schedule.

For the DOOLITTLE problem (Figures 22 and 23), the conclusions obtained with 3 processors remain true. ILHA is better than HEFT, and this becomes more visible as the communication cost increases.

For the LDMt problem (Figures 24 and 25), the conclusions are the same as for the DOOLITTLE problem.

\subsection{Comments}

Here are some synthetical comments on the experiments:

- The CPOP heuristic is not efficient for regular problems, where each node of the task graph is on a critical path: this comment holds for LAPLACE, STENCIL, and FORK-JOIN. However, CPOP leads to an efficient scheduling for LU, DOOLITTLE and LDMt.

- The BIL heuristic gives good results for LAPLACE, FORK-JOIN, DOOLITTLE and $\mathrm{LDM}^{t}$, but relatively bad ones for STENCIL and LU.

- PCT is efficient for LU, DOOLITTLE, LDMt and FORK-JOIN but it is dramatically bad for LAPLACE and STENCIL.

- GDL never gives good results, but it is never the worst heuristic.

- ILHA and HEFT give very satisfactory results.

It appears that HEFT and ILHA heuristics give very close results. However, if we consider the number of communications generated by both heuristics, we point out that our algorithm involves significantly fewer communications: see Table 1 . 


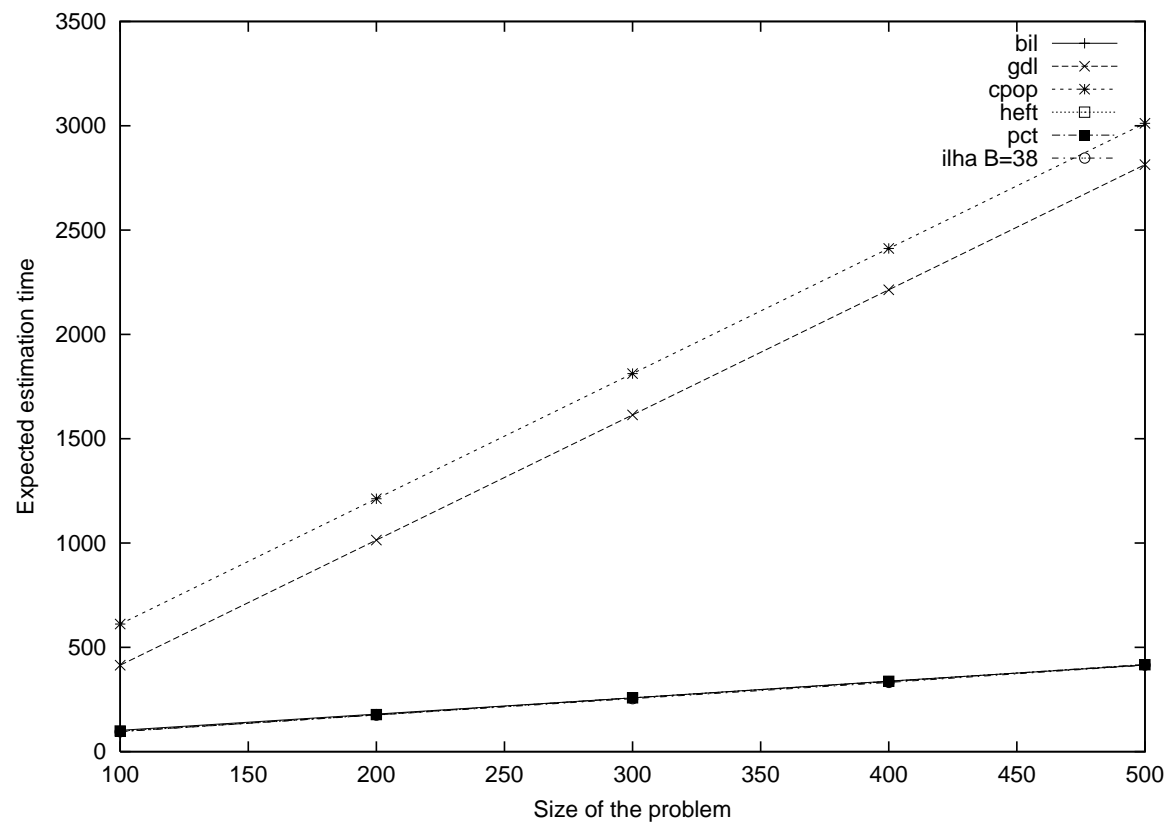

Figure 20: Comparison of the different heuristics for the FORK-JOIN problem, with 10 processors and a communication cost equal to 1.

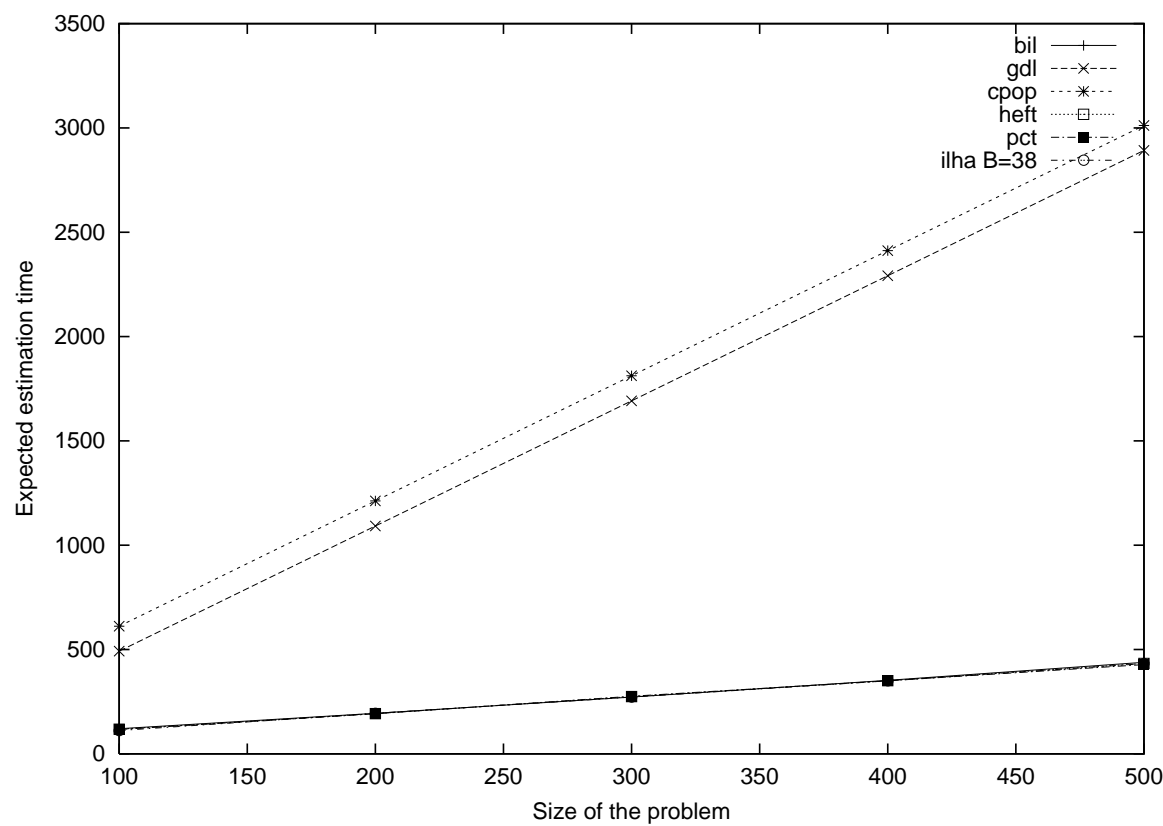

Figure 21: Comparison of the different heuristics for the FORK-JOIN problem, with 10 processors and a communication cost equal to 10 . 


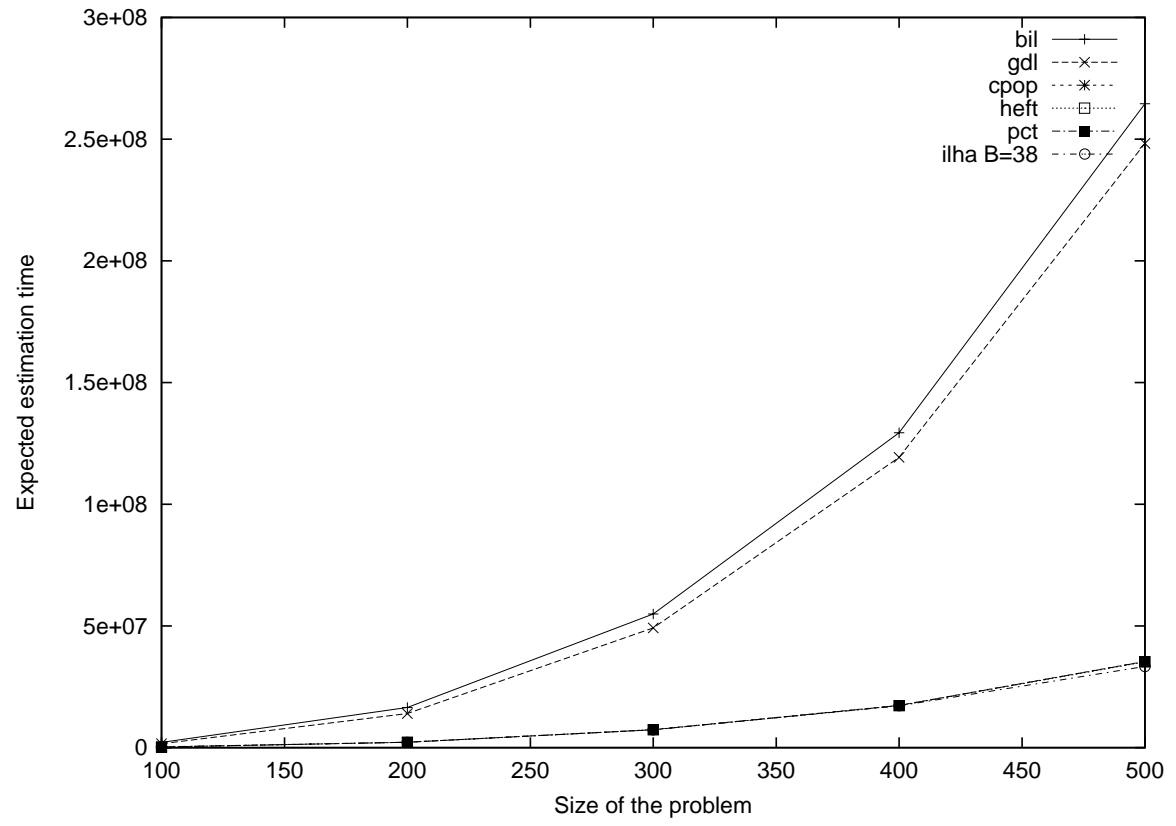

Figure 22: Comparison of the different heuristics for the DOOLITTLE problem, with 10 processors and a communication cost equal to 1.

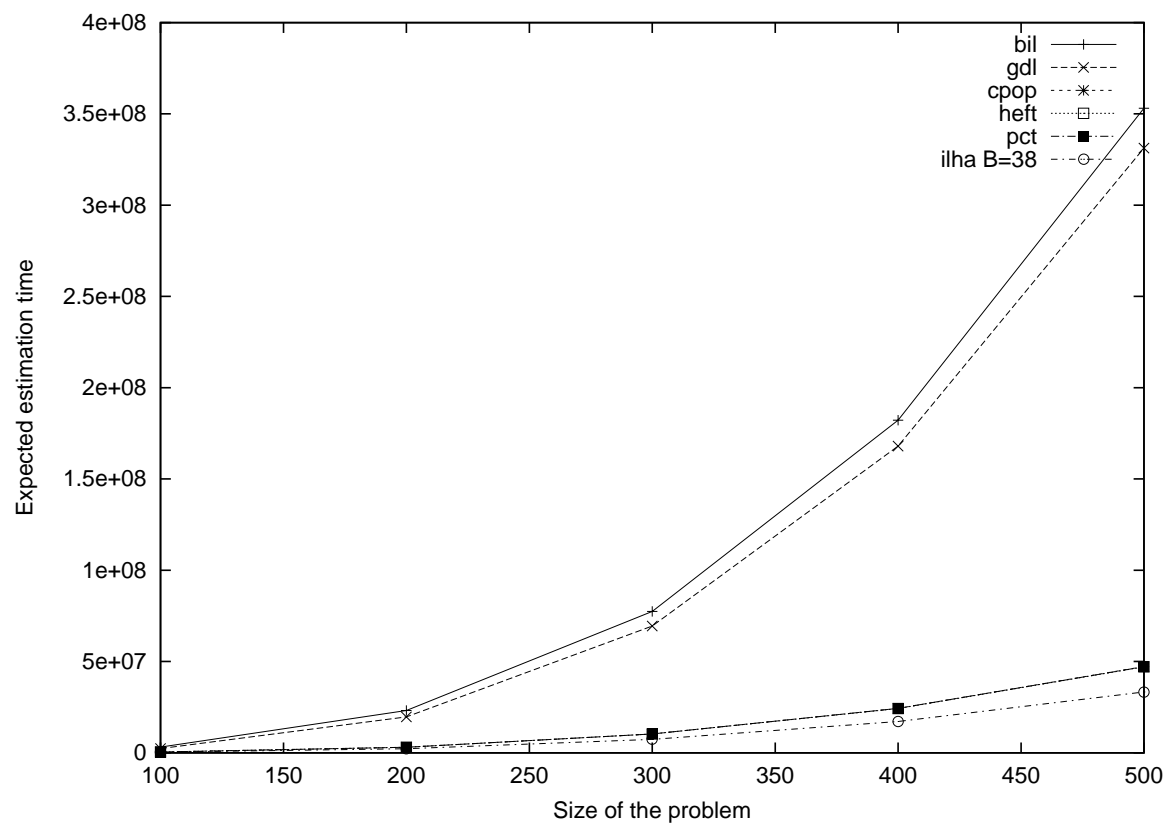

Figure 23: Comparison of the different heuristics for the DOOLITTLE problem, with 10 processors and a communication cost equal to 10 . 


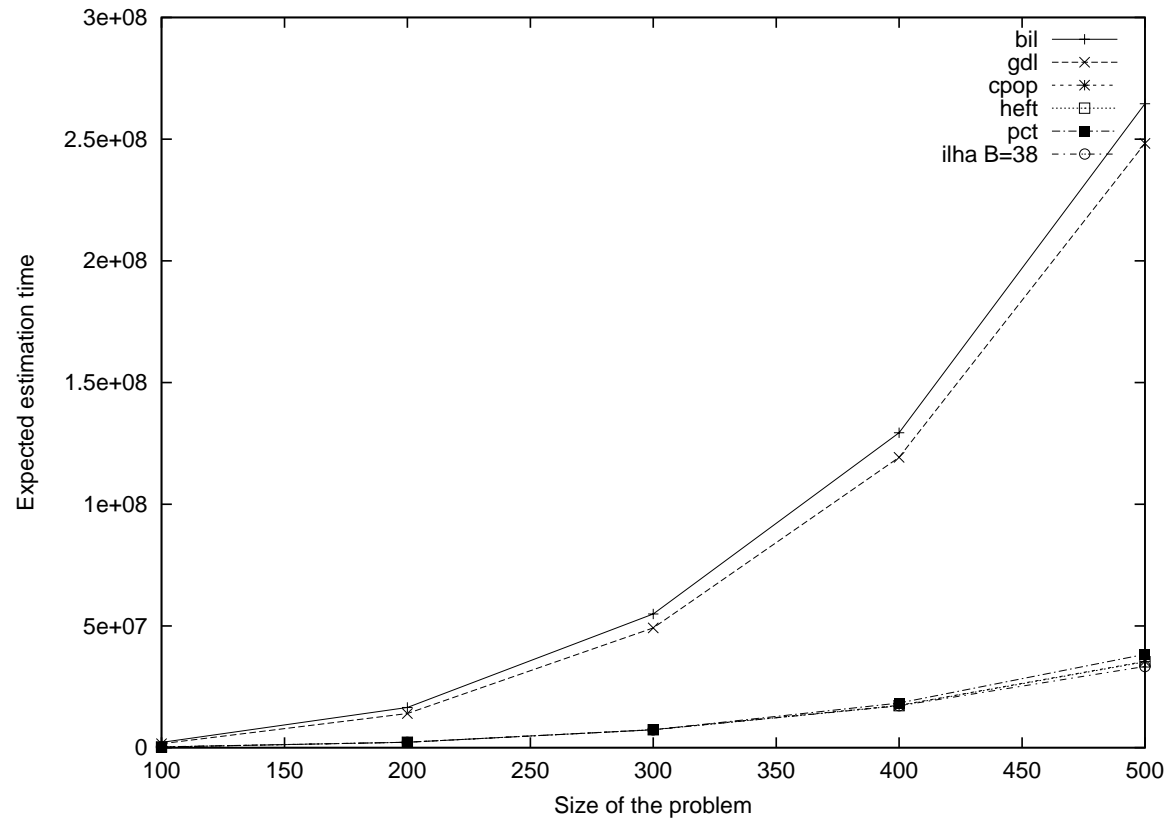

Figure 24: Comparison of the different heuristics for the LDMt problem, with 10 processors and a communication cost equal to 1.

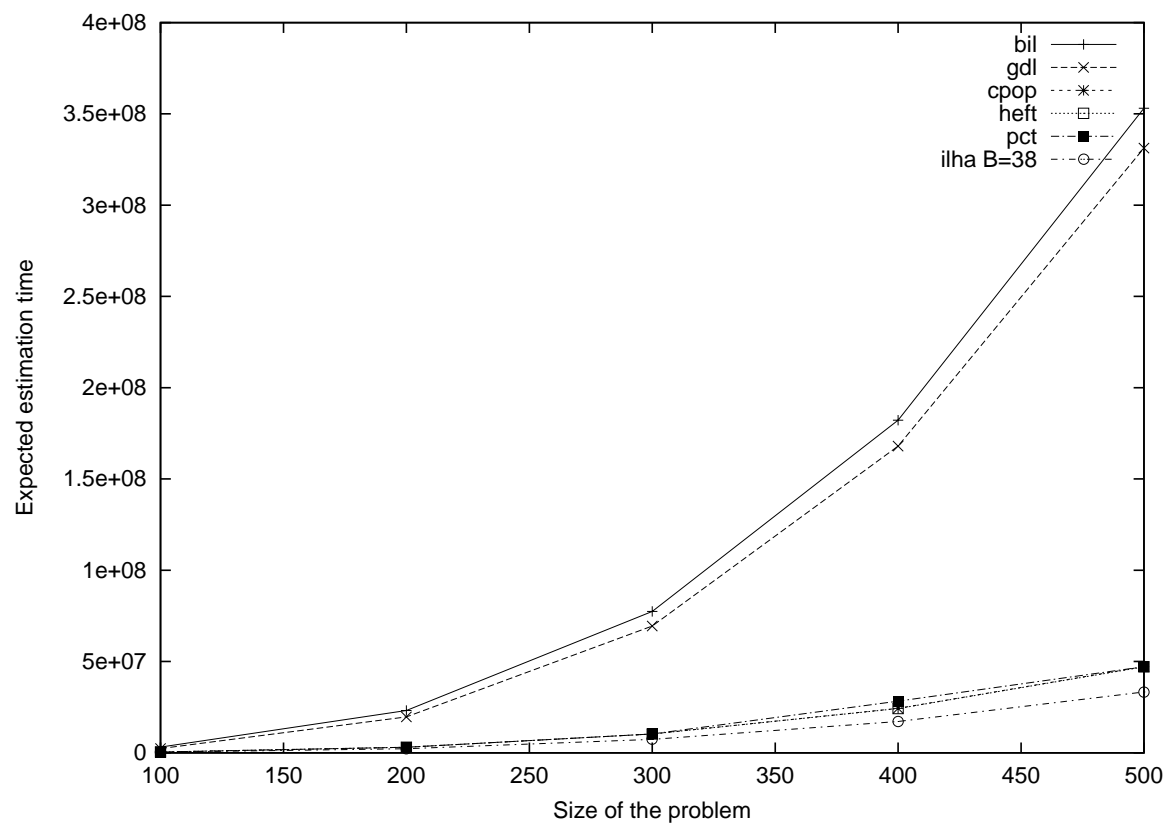

Figure 25: Comparison of the different heuristics for the LDMt problem, with 10 processors and a communication cost equal to 10 . 
In the classical scheduling model (as outlined in Section 2), we assume that the number of communications that can be performed at the same time is not limited. While this model is widely used in the scheduling community, it is not realistic in practice. The interest of ILHA may well become more important in actual implementations on heterogeneous networks of workstations, where communication resources often turn out to be the limiting factor to achieving good performances.

\begin{tabular}{|c|c|c|c|}
\hline Problem & Heft & Ilha $\mathrm{B}=10$ & Ilha $\mathrm{B}=100$ \\
\hline STENCIL & 17129 & 5742 & 396 \\
\hline LU & 11244 & 4494 & 2508 \\
\hline LAPLACE & 17129 & 8669 & 392 \\
\hline FORKJOIN & 100 & 100 & 100 \\
\hline DOOLITTLE & 13727 & 10585 & 5018 \\
\hline LDMt & 14387 & 10494 & 5272 \\
\hline
\end{tabular}

Table 1: Number of communications with HEFT and ILHA

\section{Conclusion}

In this paper we have dealt with five different heuristics from the literature to solve the task scheduling problem on heterogeneous platforms: PCT, BIL, HEFT, CPOP and GDL. It appears that HEFT is the only heuristic giving good results for all the six testbeds used in our comparison.

We have introduced a new heuristic, ILHA, which seems very promising for the following main reasons: (i) ILHA is always the best heuristic with HEFT, (ii) ILHA only requires a traversal of the task graph, so its low complexity makes its suitable to process huge task graphs in a reasonable time. (iii) ILHA generates very few communications.

\section{References}

[1] B.A.Shirazi, A.R. Hurson, and K.M. Kavi. Scheduling and load balancing in parallel and distributed systems. IEEE Computer Science Press, 1995.

[2] Vincent Boudet, Fabrice Rastello, and Yves Robert. A proposal for a heterogeneous cluster ScaLAPACK (dense linear solvers). In Hamid R. Arabnia, editor, International Conference on Parallel and Distributed Processing Techniques and Applications (PDPTA'99). CSREA Press, 1999. Extended version available as LIP Technical Report RR-99-17.

[3] P. Chrétienne, E.G. Coffman Jr., J.K. Lenstra, and Z. Liu, editors. Scheduling Theory and its Applications. John Wiley and Sons, 1995.

[4] Gene H. Golub and Charles F. Van Loan. Matrix computations. Johns Hopkins, 2 edition, 1989.

[5] M. Maheswaran and H. J. Siegel. A dynamic matching and scheduling algorithm for heterogeneous computing systems. In Seventh Heterogeneous Computing Workshop. IEEE Computer Society Press, 1998.

[6] Hyunok Oh and Soonhoi Ha. A static scheduling heuristic for heterogeneous processors. In Proceedings of Europar'96, volume 1123 of LNCS, Lyon, France, August 1996. Springer Verlag.

[7] Yves Robert, Michel Cosnard, Mounir Marrakchi, and Denis Trystram. Parallel Gaussian elimination on a MIMD computer. Parallel Computing, 6:275-296, 1988.

[8] G.C. Sih and E.A. Lee. A compile-time scheduling heuristic for interconnection-constrained heterogeneous processor architectures. IEEE Transactions on Parallel and Distributed Systems, 4(2):175-187, 1993. 
[9] H. Topcuoglu, S. Hariri, and M.-Y. Wu. Task scheduling algorithms for heterogeneous processors. In Eighth Heterogeneous Computing Workshop. IEEE Computer Society Press, 1999.

[10] Tao Yang and Apostolos Gerasoulis. DSC: Scheduling parallel tasks on an unbounded number of processors. IEEE Trans. Parallel and Distributed Systems, 5(9):951-967, 1994. 